\title{
Unilateral problem for the Stokes equations: the well-posedness and finite element approximation
}

\author{
Norikazu Saito $^{\mathrm{a}, *}$, Yoshiki Sugitani ${ }^{\mathrm{a}}$, Guanyu Zhou ${ }^{\mathrm{a}}$ \\ ${ }^{a}$ Graduate School of Mathematical Sciences, The University of Tokyo, \\ 3-8-1 Komaba, Meguro, Tokyo 153-8914, Japan
}

\begin{abstract}
We consider the stationary Stokes equations under a unilateral boundary condition of Signorini's type, which is one of artificial boundary conditions in flow problems. Well-posedness is discussed through its variational inequality formulation. We also consider the finite element approximation for a regularized penalty problem. The well-posedness, stability and error estimates of optimal order are established. The lack of a coupled Babuška and Brezzi's condition makes analysis difficult. We offer a new method of analysis. Particularly, our device to treat the pressure is novel and of some interest. Numerical examples are presented to validate our theoretical results.
\end{abstract}

Keywords: Stokes equations, finite element approximation, unilateral boundary condition 2010 MSC: 65N30, 35J87, 35Q30

\section{Introduction}

We suppose that $\Omega$ is a bounded domain in $\mathbb{R}^{d}$ with $d=2,3$ and that the boundary $\partial \Omega$ is comprises of three parts $S_{1}, S_{2}$ and $\Gamma$. Those $S_{1}, S_{2}$ and $\Gamma$ are assumed to be smooth but the whole boundary $\partial \Omega$ is not necessarily smooth. One might imagine a branched pipe resembling that depicted in Fig. 1. The first purpose of this paper is to study the well-posedness of the following unilateral boundary value problem for the Stokes equations

$$
\begin{array}{ll}
-\nu \Delta u+\nabla p=f, \quad \nabla \cdot u=0 & \text { in } \Omega, \\
u=0 & \text { on } S_{1} \cup S_{2}, \\
u_{n}+g_{n} \geq 0, & \text { on } \Gamma, \\
\tau_{n}(u, p)+\alpha_{n} \geq 0 & \text { on } \Gamma, \\
\left(u_{n}+g_{n}\right)\left(\tau_{n}(u, p)+\alpha_{n}\right)=0 & \text { on } \Gamma, \\
\tau_{T}(u)+\alpha_{T}=0 & \text { on } \Gamma
\end{array}
$$

for velocity $u=\left(u_{1}, \ldots, u_{d}\right)$ and pressure $p$ with density $\rho=1$ and kinematic viscosity $\nu$ of the viscous incompressible fluid under consideration. Therein,

$$
\tau(u, p)=\sigma(u, p) n
$$

denotes the traction vector on $\partial \Omega$, where $n$ is the outward normal vector to $\partial \Omega, \sigma(u, p)=\left(\sigma_{i j}(u, p)\right)_{1 \leq i, j \leq d}=$ $-p I+2 \nu D(u)$ the stress tensor, $D(u)=\left(D_{i j}(u)\right)_{1 \leq i, j \leq d}=\frac{1}{2}\left(\nabla u+\nabla u^{\mathrm{T}}\right)$ the deformation-rate tensor and $I$ the identity matrix. For a vector-valued function $v$ on $\partial \Omega$, its normal and tangential components are denoted, respectively, as

$$
v_{n}=v \cdot n, \quad v_{T}=v-v_{n} n .
$$

\footnotetext{
* Corresponding author

Email addresses: norikazu@ms.u-tokyo.ac.jp (Norikazu Saito), sugitani@ms.u-tokyo.ac.jp (Yoshiki Sugitani), zhoug@ms.u-tokyo.ac.jp (Guanyu Zhou)
} 


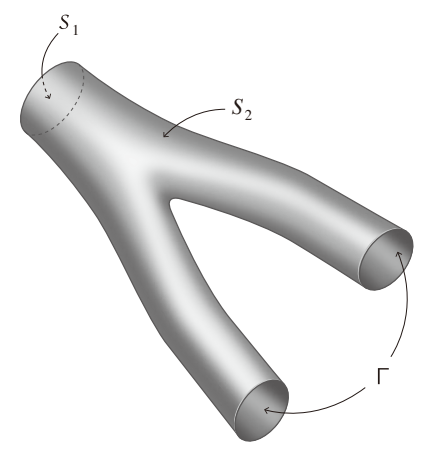

Figure 1: Example of $\Omega$ (branched pipe)

Particularly, $\tau_{n}(u, p)=\tau(u, p) \cdot n$ and $\tau_{T}(u)=\tau(u, p)-\tau_{n}(u, p) n$ respectively denote normal and tangential traction vectors. Moreover, $f, g$ and $\alpha$ are prescribed functions. We also consider the finite element approximation for a regularized penalty problem to (1) which is given as

$$
\begin{array}{ll}
-\nu \Delta u+\nabla p=f, \quad \nabla \cdot u=0 & \text { in } \Omega, \\
u=0 & \text { on } S_{1} \cup S_{2}, \\
\tau_{n}(u, p)+\alpha_{n}=\frac{1}{\varepsilon} \phi_{\delta}\left(u_{n}+g_{n}\right) & \text { on } \Gamma, \\
\tau_{T}(u)+\alpha_{T}=0 & \text { on } \Gamma
\end{array}
$$

with $0<\varepsilon \ll 1$ and $0<\delta \ll 1$. Therein, $\phi_{\delta}(s)$ is a $C^{1}$ regularization of $[s]_{-}=\max \{0,-s\}$. We can take, for example,

$$
\phi_{\delta}(s)= \begin{cases}0 & (s \geq 0) \\ \left(\sqrt{s^{2}+\delta^{2}}-\delta\right) & (s<0) .\end{cases}
$$

First, we explain our motivation for studying (1) and (2). In numerical simulation of real-world flow problems, we often encounter some issues related to artificial boundary conditions. A typical and important example is the blood flow problem in the large arteries, where the blood is assumed to be a viscous incompressible fluid (see [16,32]). The blood vessel is modeled as a branched pipe as illustrated, for example, in Fig. 1. Then, for $T>0$, we consider the Navier-Stokes equations for velocity $v=\left(v_{1}, \ldots, v_{d}\right)$ and pressure $q$,

$$
\begin{array}{ll}
v_{t}+(v \cdot \nabla) v=\nabla \cdot \sigma(v, q)+f, \quad \nabla \cdot v=0 & \text { in } \Omega \times(0, T) \\
v=b & \text { on } S_{1} \times(0, T), \\
v=0 & \text { on } S_{2} \times(0, T)
\end{array}
$$

with the initial condition $\left.v\right|_{t=0}=v_{0}$. We are able to give a velocity profile $b=b(x, t)$ at the inflow boundary $S_{1}$. The flow is presumed to be a perfect non-slip flow on the wall $S_{2}$. Then, the blood flow simulation is highly dependent on the choice of artificial boundary conditions posed on the outflow boundary $\Gamma$.

An earlier paper by Zhou and Saito [34] presented discussion of the free-traction condition

$$
\tau(v, q)=0 \quad \text { on } \quad \Gamma,
$$

which is one of the common outflow boundary conditions (see [18, 21]), and some nonlinear energypreserving boundary conditions (see $[3,7,8,11,12]$ ) from the view-point of energy inequality. Moreover, we proposed a new outflow boundary condition as

$$
v_{n} \geq 0, \quad \tau_{n}(v, q) \geq 0, \quad v_{n} \tau_{n}(v, q)=0, \quad \tau_{T}(v)=0 \quad \text { on } \Gamma .
$$

This is an analogy to Signorini's condition in the theory of elasticity (see [24]). It is indeed a generalization of the free-traction condition (5), as

$$
\begin{aligned}
& \text { if } v_{n}>0 \text { on } \omega \subset \Gamma \text {, then } \tau_{n}(v, q)=0 \text { on } \omega \text {; } \\
& \text { if } v_{n}=0 \text { on } \omega \subset \Gamma \text {, then } \tau_{n}(v, q) \geq 0 \text { on } \omega .
\end{aligned}
$$


A benefit of using (6) is that (4) admits energy inequality, although it is not guaranteed under (5). To describe it more specifically, we take a reference flow $(g, \pi)$, which is the solution of the Stokes system

$$
\begin{gathered}
\nabla \cdot \sigma(g, \pi)=0, \quad \nabla \cdot g=0 \quad \text { in } \Omega, \\
g=b \text { on } S_{1}, \quad g=0 \text { on } S_{2}, \quad g=-g_{0}(x) \int_{S_{1}} b \cdot n d S_{1} \text { on } \Gamma
\end{gathered}
$$

for all $t \in[0, T]$, where $g_{0}=g_{0}(x) \in C_{0}^{\infty}(\Gamma)^{d}$ is a prescribed function satisfying

$$
\int_{\Gamma} g_{0} \cdot n d \Gamma=1, \quad g_{0} \cdot n \geq 0 \text { on } \Gamma .
$$

(Function $g$ is nothing but a lifting function of $b$.) Using this, we will find $(v, q)$ of the form

$$
v=u+g, \quad q=p+\pi
$$

Assuming that (4) admits a smooth solution $(v, q)=(u+g, p+\pi)$ in $0 \leq t \leq T$ and multiplying both sides of (4a) by $u$, by the integration by parts, we have

$$
\begin{gathered}
\frac{d}{d t}\|u\|_{L^{2}(\Omega)^{d}}^{2}+\underbrace{2 \nu \int_{\Omega} D_{i j}(u) D_{i j}(u) d x+\frac{1}{2} \int_{\Gamma} v_{n}|u|^{2} d \Gamma-\int_{\Gamma} \tau(v, q) \cdot u d \Gamma}_{=I} \\
=\int_{\Omega}\left[f-g_{t}-(g \cdot \nabla) g\right] \cdot u d x-\int_{\Omega}(u \cdot \nabla) g \cdot u d x .
\end{gathered}
$$

Using (6), we derive $I \geq 0$. Consequently,

$$
\sup _{t \in[0, T]}\|u\|_{L^{2}(\Omega)^{d}}^{2}+2 \nu \int_{0}^{T} D_{i j}(u) D_{i j}(u) \leq C,
$$

where $C$ denotes a positive constant depending only on $f, u_{0}, b$ and $T$ (see [34, Theorem 4]). This inequality is useful. It plays a crucial role in the construction of a solution of the Navier-Stokes equations (see [34]). Moreover, it is connected with the stability of numerical schemes from the view-point of numerical computation. That is, it is preferred that energy inequality not be spoiled after discretizations (see [33]). With (5), we do not know whether $I \geq 0$ or not. Therefore, energy inequality cannot be derived even for the continuous case.

There are a lot of strategies to treat the inequality condition (6) in numerical calculations. For example, we can use its penalty approximation

$$
\tau_{n}(v, q)=\frac{1}{\varepsilon}\left[v_{n}\right]_{-}, \quad \tau_{T}(v)=0 \quad \text { on } \Gamma,
$$

where $0<\varepsilon \ll 1$ and

$$
[s]_{ \pm}=\max \{0, \pm s\}, \quad s=[s]_{+}-[s]_{-} \quad(s \in \mathbb{R}) .
$$

We also obtain energy inequality with (11) for a sufficiently small $\varepsilon$ (see [34, Theorem 5]). Moreover, after introducing a $C^{1}$ regularization $\phi_{\delta}$ of $[\cdot]_{-}$, we can solve (4) with (11) by using, for example, Newton's iteration.

Our ultimate objective is to develop the theory for the initial-boundary value problems for the NavierStokes equations (4) with (6) or with (11) from the dual standpoints of analysis and numerical computations. As a primary step, we studied the well-posedness of these problems in Ladyzhenskaya's class in [34]. That is, we studied the unique existence of a solution of

$$
\begin{array}{ll}
u_{t}+((u+g) \cdot \nabla) u+(u \cdot \nabla) g-\nabla \cdot \sigma(u, p)=F, \quad \nabla \cdot u=0 & \text { in } \Omega, \\
u=0 & \text { on } S_{1} \\
u_{n}+g_{n} \geq 0, \quad \tau_{n}(u, p)+\tau_{n}(g, \pi) \geq 0 & \text { on } \Gamma, \\
\left(u_{n}+g_{n}\right)\left(\tau_{n}(u, p)+\tau_{n}(g, \pi)\right)=0, \quad \tau_{T}(u)+\tau_{T}(g) \geq 0 & \text { on } \Gamma,
\end{array}
$$


where $F=f-g_{t}-(g \cdot \nabla) g$.

For the analyses described herein, we devote our attention to the discretization of the space variable. Therefore, we study the finite element approximation using model Stokes problems. Consequently, we come to consider Problems (1) and (2).

As a matter of fact, (1) and (2) themselves are not new problems (see $[4,13,15,24]$ for example). In a classical monograph [24], Chapter 7 is devoted to similar problems, say Signorini's problem for incompressible materials. However, their problem includes the traction condition $\tau(u, p)=h$. More precisely, they assume that $S_{2}$ is divided into two parts $S_{21}, S_{22}$ and consider

$$
u=0 \quad \text { on } S_{21}, \quad \tau(u, p)=h \quad \text { on } S_{22}
$$

instead of (1b). Then, assuming

$$
\bar{\Gamma} \cap \overline{\left(S_{1} \cup S_{21}\right)}=\emptyset,
$$

we can prove that there exists a domain constant $C>0$ satisfying

$$
C\left[\|q\|_{L^{2}(\Omega)}+\|\tau\|_{H^{-\frac{1}{2}(\Gamma)}}\right] \leq \sup _{v \in H^{1}(\Omega)^{d},\left.v\right|_{S_{1} \cup S_{21}}=0} \frac{\int_{\Omega} q(\nabla \cdot v) d x+\int_{\Gamma} \tau v_{n} d \Gamma}{\|v\|_{H^{1}(\Omega)}}
$$

for any $(q, \tau) \in L^{2}(\Omega) \times H^{-\frac{1}{2}}(\Gamma)$ (see [24, Theorem 7.2]). This inequality is usually designated as the coupled Babuška-Brezzi condition. The well-posedness and error estimates of the corresponding penalty problem (with no regularization) are direct consequences of this result from the general theory (see [5]). $[13,15]$ study similar unilateral problems and they also assume $S_{22} \neq \emptyset$. In contrast, we are interested in establishing a formulation without the traction boundary condition. Unfortunately, if $S_{22}=\emptyset$, then (14) is not available. It makes analysis somewhat difficult. Moreover, we do not prefer assuming (13). Consequently, we must develop a completely new method of analysis in this paper. Particularly, we offer a new device to treat the pressure part. As a result, we succeed in deriving the optimal-order error esimate for the finite element approximation for the penalty problem using the MINI (P1b/P1) element (see Thereom 18). Our method is different from that of [15] in which the optimal-order error estimates are proved for linear and quadratic finite element approximations for Signorini's contact problem (with no penalization). We first derive non-optimal error estimates and then apply them to improve several estimates. Our error estimates are novel even in the literature of elasticity theory (see [13]).

Finite element approximation of another class of unilateral boundary value problems for the Stokes equations, say unilateral problems of friction type, are discussed, for example, in several reports of the literature $[1,23,25,26]$.

This paper is composed of 7 sections. After introducing basic definitions and recalling some standard results in Section 2, we state two variational formulations, (PDE) and (VI), for (1) in Section 3. The equivalence of these formulations and well-posedness of (VI) are also established there (see Theorems 5 and 6). Section 4 is devoted to presentation of the finite element approximation for (2). We consider only the MINI (P1b/P1) element approximation. The well-posedness and error estimates are proved, respectively, in Sections 5 and 6 (see Theorems 9, 10 and 18). Finally, we confirm our results by numerical experiments in Section 7.

\section{Preliminaries}

Geometry. We recall that $\Omega \subset \mathbb{R}^{d}, d=2,3$, is a bounded domain and the boundary $\partial \Omega$ comprises of three parts $S_{1}, S_{2}$ and $\Gamma$. We address the following two cases:

(G1) $S_{1}, S_{2}$ and $\Gamma$ are smooth surfaces (curves) and $\Omega$ is a Lipschitz domain (see Fig. 1);

(G2) $S_{1}, S_{2}$ are polygon (line segment) and $\Omega$ is a polyhedral (polygonal) domain.

For the following, we assume that $\Omega$ is given as (G1) or (G2) unless otherwise stated explicitly. Moreover, we set

$$
S=S_{1} \cup S_{2}
$$

and assume that the $d-1$ dimensional Lebesgue measure $|S|$ is positive.

Throughout this paper, $C$ denotes various positive constants depending on $\Omega$. 
Remark 1. Although we mostly address the case presented in Fig. 1, our discussion is also valid for the case in which $\partial \Omega$ is smooth with $\bar{\Gamma} \cap \bar{S}_{2}=\emptyset, \bar{S}_{2} \cap \bar{S}_{1}=\emptyset$, and $\bar{S}_{1} \cap \bar{\Gamma}=\emptyset$.

Function spaces and forms. We use the standard Lebesgue and Sobolev spaces, for example, $L^{2}(\Omega), H^{1}(\Omega)$, $L^{2}(\Gamma), H^{\frac{1}{2}}(\Gamma)$. (We follow the notation of [29] as for function spaces and their norms. ) The abbreviations

$$
\begin{gathered}
(v, w)=(v, w)_{\Omega}=(v, w)_{L^{2}(\Omega)}, \quad(v, w)_{\Gamma}=(v, w)_{0, \Gamma}=(v, w)_{L^{2}(\Gamma)}, \\
\|v\|=\|v\|_{\Omega}=\|v\|_{0, \Omega}=\|v\|_{L^{2}(\Omega)}, \quad\|v\|_{1}=\|v\|_{1, \Omega}=\|v\|_{H^{1}(\Omega)}, \quad\|v\|_{\Gamma}=\|v\|_{0, \Gamma}=\|v\|_{L^{2}(\Gamma)}
\end{gathered}
$$

will be used. Moreover,

$$
|v|_{m}=|v|_{m, \Omega}=|v|_{H^{m}(\Omega)}, \quad|v|_{m, \Gamma}=|v|_{H^{m}(\Gamma)}
$$

are the semi-norms of $H^{m}(\Omega), H^{m}(\Gamma)$.

For a vector-valued function space, we use the same symbol to denote its norm as

$$
\|v\|=\|v\|_{L^{2}(\Omega)^{d}} \quad\left(v \in L^{2}(\Omega)^{d}\right), \quad\|v\|_{1}=\|v\|_{H^{1}(\Omega)^{d}} \quad\left(v \in H^{1}(\Omega)^{d}\right) .
$$

The basic function spaces of our consideration are

$$
V=\left\{v \in H^{1}(\Omega)^{d} \mid v=0 \text { on } S\right\} \text { and } Q=L^{2}(\Omega) .
$$

They are, respectively, Hilbert spaces equipped with the norms $\|v\|_{1}$ and $\|q\|$. We use closed subspaces of V,

$$
V^{\sigma}=\{v \in V \mid \nabla \cdot v=0 \text { in } \Omega\}, \quad V_{0}=H_{0}^{1}(\Omega)^{d}, \quad V_{0}^{\sigma}=\left\{v \in V_{0} \mid \nabla \cdot v=0 \text { in } \Omega\right\},
$$

and that of $Q$ as

$$
Q_{0}=\left\{q \in Q \mid \int_{\Omega} q d x=0\right\}
$$

Convex subsets

$$
K=\left\{v \in V \mid v_{n}+g_{n} \geq 0 \text { on } \Gamma\right\} \quad \text { and } \quad K^{\sigma}=\left\{v \in V^{\sigma} \mid v_{n}+g_{n} \geq 0 \text { on } \Gamma\right\}
$$

of $V$ and $V^{\sigma}$, respectively, play important roles.

We recall the so-called Lions-Magenes space $H_{00}^{\frac{1}{2}}(\Gamma)$. It is defined as (see $[29, \S 11.5$, Ch. 1])

$$
H_{00}^{\frac{1}{2}}(\Gamma)=\left\{\mu \in H^{\frac{1}{2}}(\Gamma) \mid \rho^{-1 / 2} \mu \in L^{2}(\Gamma)\right\}
$$

which is a Hilbert space equipped with the norm

$$
\|\mu\|_{H_{00}^{\frac{1}{2}(\Gamma)}}=\left(\|\mu\|_{H^{\frac{1}{2}}(\Gamma)}^{2}+\left\|\rho^{-1 / 2} \mu\right\|_{\Gamma}^{2}\right)^{\frac{1}{2}} .
$$

Here, $\rho \in C^{\infty}(\bar{\Gamma})$ denotes any positive function satisfying $\left.\rho\right|_{\partial \Gamma}=0$, and for $x_{0} \in \partial \Gamma$,

$$
\lim _{x \rightarrow x_{0}} \frac{\rho(x)}{\operatorname{dist}(x, \partial \Gamma)}=d^{\prime}>0
$$

with some $d^{\prime}>0$. Moreover, we know (see [29, Theorem 11.7, Ch. 1])

$$
H_{00}^{\frac{1}{2}}(\Gamma)=\left(H_{0}^{1}(\Gamma), L^{2}(\Gamma)\right)_{\frac{1}{2}, 2} \quad \text { (algebraically and topologically), }
$$

where the right-hand side denotes the real interpolation space between $L^{2}(\Gamma)$ and $H_{0}^{1}(\Gamma)$ with the exponent $1 / 2$ and $p=2$. Particularly $H_{00}^{\frac{1}{2}}(\Gamma)$ is strictly included in $H^{\frac{1}{2}}(\Gamma)$.

Below we set

$$
M=H_{00}^{\frac{1}{2}}(\Gamma), \quad\|\mu\|_{\frac{1}{2}, \Gamma}=\|\mu\|_{H_{00}^{\frac{1}{2}}(\Gamma)}
$$


and

$$
M_{0}=\left\{\mu \in M \mid \int_{\Gamma} \mu d \Gamma=0\right\} .
$$

In general, $X^{\prime}$ denotes the topological dual space of a Banach space $X$. The norm of $X^{\prime}$ is defined as

$$
\|\varphi\|_{X^{\prime}}=\sup _{v \in X, v \neq 0} \frac{\langle\varphi, v\rangle_{X^{\prime}, X}}{\|v\|_{X}}
$$

where $\langle\cdot, \cdot\rangle_{X^{\prime}, X}$ is the duality pairing between $X^{\prime}$ and $X$. For a closed subspace $Y$ of $X$ and $\varphi \in Y^{\prime}$, we mean by $\|\varphi\|_{Y^{\prime}}$

$$
\|\varphi\|_{Y^{\prime}}=\sup _{v \in Y, v \neq 0} \frac{\langle\varphi, v\rangle_{X^{\prime}, X}}{\|v\|_{X}} .
$$

Set

$$
\begin{gathered}
\langle\cdot, \cdot\rangle=\langle\cdot, \cdot\rangle_{V^{\prime}, V}=\text { the duality pairing between } V^{\prime} \text { and } V \\
{[\cdot, \cdot]=[\cdot, \cdot]_{M^{\prime}, M}=\text { the duality pairing between } M^{\prime} \text { and } M,} \\
{[[\cdot, \cdot]]=[[\cdot, \cdot]]_{\left(M^{d}\right)^{\prime}, M^{d}}=\text { the duality pairing between }\left(M^{d}\right)^{\prime} \text { and } M^{d} .}
\end{gathered}
$$

We use the following forms:

$$
\begin{aligned}
& a(u, v)=2 \nu \int_{\Omega} D_{i j}(u) D_{i j}(v) d x \quad\left(u, v \in H^{1}(\Omega)^{d}\right) ; \\
& b(p, u)=-\int_{\Omega} p(\nabla \cdot u) d x \quad\left(p \in Q, u \in H^{1}(\Omega)^{d}\right) .
\end{aligned}
$$

Trace and lifting operators. Let $\operatorname{Tr}=\operatorname{Tr}(\Omega, \Gamma)$ be a trace operator from $H^{1}(\Omega)$ into $H^{\frac{1}{2}}(\Gamma)$. The meaning of $\operatorname{Tr}(\Omega, S)$ is the same.

Lemma 2. The trace operator $v \mapsto \mu=\operatorname{Tr} v$ is linear and continuous of $V \rightarrow M^{d}$. Conversely, there exists a linear and bounded operator $\mathcal{E}$ of $M^{d} \rightarrow V$, which is called a lifting operator, such that $\mathcal{E} \mu=\mu$ on $\Gamma$ for all $\mu \in M^{d}$.

This result follows directly from [19, Theorem 2.5] and [20, Theorem 1.5.2.3]. A partial result is also reported in [30, Theorems 1.1 and 5.1]. As a consequence of Lemma 2, we obtain a lifting operator $\mathcal{E}_{n}: M \rightarrow V$ such that

$$
\left(\mathcal{E}_{n} \mu\right)_{n}=\mu, \quad\left(\mathcal{E}_{n} \mu\right)_{T}=0 \text { on } \Gamma, \quad\left\|\mathcal{E}_{n} \mu\right\|_{1} \leq C\|\mu\|_{\frac{1}{2}, \Gamma}
$$

for any $\mu \in M$.

Below, we will often write as $\left.v\right|_{\Gamma}=\operatorname{Tr} v$ if there is no fear of confusion.

Remark 3. In view of Lemma 2 and the standard trace/lifting theorem, the zero extension $\hat{\mu}$ of $\mu \in M^{d}$ into $\partial \Omega$;

$$
\hat{\mu}= \begin{cases}\mu & \text { on } \Gamma \\ 0 & \text { on } \partial \Omega \backslash \Gamma\end{cases}
$$

belongs to $H^{\frac{1}{2}}(\partial \Omega)^{d}$.

Remark 4. Another definition of $H_{00}^{\frac{1}{2}}(\Gamma)$ is given by Baiocchi and Capelo [2, Page 379]. That is,

$$
H_{00}^{\frac{1}{2}}(\Gamma)=\left\{\operatorname{Tr} v \mid v \in H^{1}(\Omega), \operatorname{Tr}(\Omega, S) v=0\right\}
$$

which is a Hilbert space equipped with the norm

$$
\|\mu\|_{H_{00}^{\frac{1}{2}}(\Gamma)}=\inf \left\{\|v\|_{1} \mid v \in H^{1}(\Omega), \operatorname{Tr}(\Omega, S) v=0, \operatorname{Tr} v=\mu\right\} .
$$


Redefinition of traction vectors. Next we propose the redefinition of $\tau(u, p)$. If a smooth vector field $u$ and scalar field $p$ satisfy the Stokes equation

$$
-\nu \Delta u+\nabla p=f, \quad \nabla \cdot u=0 \quad \text { in } \Omega
$$

for a given $f \in L^{2}(\Omega)^{d}$, they satisfy

$$
a(u, v)+b(p, v)+\int_{\Gamma} \tau(u, p) \cdot v=(f, v) \quad(\forall v \in V)
$$

and

$$
a(u, v)+b(p, v)=(f, v) \quad\left(\forall v \in V_{0}\right) .
$$

(In (16), $\tau(u, p)$ is understood as a usual function defined on $\Gamma$.) Based on those identities, for functions $(u, p) \in V^{\sigma} \times Q$ satisfying (17), we redefine the traction vector $\tau(u, p)$ as a functional over $M^{d}$ by

$$
[[\tau(u, p), \mu]]=a\left(u, w_{\mu}\right)+b\left(p, w_{\mu}\right)-\left(f, w_{\mu}\right) \quad\left(\mu \in M^{d}\right),
$$

where $w_{\mu}=\mathcal{E} \mu \in V$. Actually, the right-hand side of (18) is independent of the way of extension. Therefore, this definition is well-defined. Similarly, we redefine

$$
\left[\left[\tau_{T}(u), \mu\right]\right]=a\left(u, w_{\mu}\right)+b\left(p, w_{\mu}\right)-\left(f, w_{\mu}\right) \quad\left(\mu \in M^{d} \text { with } \mu_{n}=0 ; w_{\mu}=\mathcal{E} \mu\right)
$$

and

$$
\left[\tau_{n}(u, p), \mu\right]=a\left(u, w_{\mu}\right)+b\left(p, w_{\mu}\right)-\left(f, w_{\mu}\right) \quad\left(\mu \in M ; w_{\mu}=\mathcal{E}_{n} \mu\right) .
$$

Then, we deduce an expression

$$
[[\tau(u, p), \mu]]=\left[\tau_{n}(u, p), \mu_{n}\right]+\left[\left[\tau_{T}(u), \mu_{T}\right]\right] \quad\left(\mu \in M^{d}\right) .
$$

\section{Variational formulations and well-posedness}

From this point forward in our discussion, we always assume

$$
f \in Q^{d}, \quad b \in M^{d}, \quad \beta \equiv-\int_{S} b \cdot n d S>0 .
$$

We take $g \in H^{1}(\Omega)^{d}$ satisfying

$$
\nabla \cdot g=0 \text { in } \Omega,\left.\quad g\right|_{S}=b,\left.\quad g\right|_{C}=0,\left.\quad g\right|_{\Gamma}=\beta g_{0},
$$

where $g_{0}$ is the function defined as (8). Then, we have

$$
g_{n} \geq 0 \text { on } \Gamma, \quad g_{n} \in M, \quad \alpha \equiv 2 \nu D(g) n \in\left(M^{d}\right)^{\prime}
$$

Under those assumptions and redefinitions in the previous section, we interpret (1) precisely as follows. (PDE) Find $(u, p) \in V \times Q$ such that

$$
\begin{array}{ll}
a(u, v)+b(p, v)=(f, v) & \left(\forall v \in V_{0}\right), \\
b(q, u)=0 & (\forall q \in Q), \\
u_{n}+g_{n} \geq 0 & \text { a.e. on } \Gamma, \\
{\left[\tau_{n}(u, p)+\alpha_{n}, \mu\right] \geq 0} & (\forall \mu \in M, \mu \geq 0), \\
{\left[\tau_{n}(u, p)+\alpha_{n}, u_{n}+g_{n}\right]=0} & \\
{\left[\left[\tau_{T}(u)+\alpha_{T}, \mu\right]\right]=0} & \left(\forall \mu \in M^{d}, \mu_{n}=0\right) .
\end{array}
$$

If a solution $(u, p)$ of (PDE) is sufficiently smooth, it solves (1) in the classical sense. Actually, (PDE) is equivalent to the following variational inequality.

(VI) Find $(u, p) \in K \times Q$ such that

$$
\begin{array}{ll}
a(u, v-u)+b(p, v-u) \geq(f, v-u)-[[\alpha, v-u]] & (\forall v \in K), \\
b(q, u)=0 & (\forall q \in Q) .
\end{array}
$$

In this section, we prove the following two theorems. 
Theorem 5. Problems (VI) and (PDE) are equivalent.

Theorem 6. There exists a unique solution $(u, p) \in K \times Q$ of (VI) and it holds that

$$
\|u\|_{1}+\|p\| \leq C_{*},
$$

where $C_{*}$ denotes a positive constant depending only on $\Omega,\|f\|,\|\alpha\|_{\left(M^{d}\right)^{\prime}}$ and $\|g\|_{1}$.

Remark 7 . The boundary condition (22f) is nothing but one alternative. One can pose

$$
u_{T}+\alpha_{T}^{\prime}=0 \quad \text { a.e. on } \Gamma
$$

with a prescribed $\alpha_{T}^{\prime}$ instead of (22f). Actually, the discussion presented below remains true if we re-choose a suitable lifting function $g$ and replace the original $V$ with

$$
V=\left\{v \in H^{1}(\Omega)^{d} \mid v=0 \text { on } \Gamma, v_{T}=0 \text { on } \Gamma\right\} .
$$

Proof of Theorem 5. (PDE) $\Rightarrow(\mathrm{VI})$. Let $(u, p) \in V \times Q$ be a solution of (PDE). We verify $(u, p)$ is a solution of (VI). First, we have $u \in K$ by (22c) and (22b). By using (18), (21), and (22c)-(22f), we have for any $v \in K$

$$
\begin{aligned}
& a(u, v-u)+b(p, v-u)-(f, v-u)+[[\alpha, v-u]] \\
& =[[\tau(u, p), v-u]]+[[\alpha, v-u]] \\
& =\left[\tau_{n}(u, p), v_{n}-u_{n}\right]+\underbrace{\left[\left[\tau_{T}(u)+\alpha_{T}, v_{T}-u_{T}\right]\right]}_{=0}+\left[\alpha_{n}, v_{n}-u_{n}\right] \\
& =\underbrace{\left[\tau_{n}(u, p)+\alpha_{n}, v_{n}+g_{n}\right]}_{\geq 0}-\underbrace{\left[\tau_{n}(u, p)+\alpha_{n}, u_{h}+g_{n}\right]}_{=0} \geq 0 .
\end{aligned}
$$

$(\mathrm{VI}) \Rightarrow(\mathrm{PDE})$. Let $(u, p) \in K \times Q$ be a solution of (VI). We now verify $(u, p)$ actually satisfies (PDE). First, (22b) and (22c) are obvious.

Let $v \in V_{0}$ be arbitrary. Substituting $v=u \pm v \in K$ into (23a), we have (22a).

We recall $\tau(u, p)$ is defined as (18). Consequently, (23a) implies

$$
[[\tau(u, p), v-u]] \geq-[[\alpha, v-u]] \quad(\forall v \in K) .
$$

Moreover, by (21)

$$
\left[\tau_{n}(u, p)+\alpha_{n}, v_{n}-u_{n}\right]+\left[\left[\tau_{T}(u)+\alpha_{T}, v_{T}-u_{T}\right]\right] \geq 0 \quad(\forall v \in K) .
$$

Let $\psi \in C_{0}^{\infty}(\Gamma)^{d}$ with $\psi_{n}=0$ on $\Gamma$. Substituting $v=u \pm \mathcal{E} \psi \in K$ into (26), we have $\left[\left[\tau_{T}(u)+\alpha_{T}, \psi\right]\right]=0$. By density, this implies (22f).

Next, let $\psi \in C_{0}^{\infty}(\Gamma)^{d}$ with $\psi_{n} \geq 0$ and $\psi_{T}=0$ on $\Gamma$. Substituting $v=u+\mathcal{E} \psi \in K$ into (26), we have $\left[\tau_{n}(u, p)+\alpha_{n}, \psi_{n}\right] \geq 0$. By density, this implies $(22 \mathrm{~d})$.

Combining (26) and (22f), we have

$$
\left[\tau_{n}(u, p)+\alpha_{n}, v_{n}-u_{n}\right] \geq 0 \quad(\forall v \in K) .
$$

At this stage, we introduce $w^{*} \in V$ satisfying

$$
w^{*}=g \text { on } \Gamma, \quad\left\|w^{*}\right\|_{1} \leq C\|g\|_{1} .
$$

Since $\left.g\right|_{\Gamma} \in M^{d}$, such $w^{*}$ truly exists in view of the trace theorem. However, it does not satisfy the divergence-free condition. Consequently, $w^{*} \notin V^{\sigma}$. We now have $-w_{n}^{*}+g_{n}=-g_{n}+g_{n} \geq 0$ and $2 u_{n}+w_{n}^{*}+g_{n}=2\left(u_{n}+g_{n}\right) \geq 0$. Therefore, we can choose as $v=-w^{*}$ and $v=2 u+w^{*}$ above and obtain (22e). 
Proof of Theorem 6. Since $a$ is a coercive bilinear form in $V^{\sigma} \times V^{\sigma}$ by virtue of Korn's inequality (see [24, Lemma 6.2]), we can apply Stampacchia's theorem (see [10, Theorem 5.6]) to conclude that there exists a unique $u \in K^{\sigma}$ satisfying the following:

$$
a(u, v-u) \geq(f, v-u)-[[\alpha, v-u]] \quad\left(\forall v \in K^{\sigma}\right) .
$$

Taking $v=u \pm \varphi$ with $\varphi \in V_{0}^{\sigma}$ in (28), we deduce

$$
a(u, \varphi)=(f, \varphi) \quad\left(\forall \varphi \in V_{0}^{\sigma}\right) .
$$

Therefore, according to [17, Lemma I.2.1], there exists a unique $\hat{p} \in Q_{0}$ satisfying

$$
-b(\hat{p}, v)=a(u, v)-(f, v) \quad\left(\forall v \in V_{0}\right) .
$$

Now we set, for $k \in \mathbb{R}$,

$$
p_{k}=\hat{p}+k
$$

and verify, with an appropriate choice of $k$, that $\left(u, p_{k}\right)$ is a solution of (VI). To this end, it suffices to check that $\left(u, p_{k}\right)$ is a solution of (PDE).

We have by (18) and (28)

$$
\left[\tau_{n}\left(u, p_{k}\right), v_{n}-u_{n}\right]+\left[\left[\tau_{T}(u), v_{T}-u_{T}\right]\right] \geq-[[\alpha, v-u]] \quad\left(v \in K^{\sigma}\right) .
$$

Letting $\psi \in C_{0}^{\infty}(\Gamma)^{d}$ with $\psi_{n}=0$ on $\Gamma$, then since $\int_{\Gamma} \psi_{n} d \Gamma=0$, there is a function $w \in V$ satisfying $w=\psi$ on $\Gamma, \nabla \cdot w=0$ in $\Omega$ and $\|w\|_{1} \leq C\|\psi\|_{M^{d}}$. Substituting $v=u \pm w \in K^{\sigma}$ into (32), one obtains $\left[\left[\tau_{T}(u)+\alpha_{T}, \psi_{T}\right]\right]=0$. By density, this implies (22f).

Therefore, it follows from (32) that

$$
\left[\tau_{n}\left(u, p_{k}\right)+\alpha_{n}, v_{n}-u_{n}\right] \geq 0 \quad\left(v \in K^{\sigma}\right) .
$$

We set

$$
\gamma=\inf _{\mu \in Y}\left[\tau_{n}(u, \hat{p})+\alpha_{n}, \mu\right]
$$

where

$$
Y=\left\{\mu \in M \mid \mu \geq 0, \mu \not \equiv 0, \int_{\Gamma} \mu d \Gamma=1\right\} .
$$

For any $\mu \in M$ with $\mu \geq 0$ and $\mu \not \equiv 0$, we have

$$
\left[\tau_{n}\left(u, p_{k}\right)+\alpha_{n}, \mu\right]=\left[\tau_{n}(u, \hat{p})+\alpha_{n}, \mu\right]-k \int_{\Gamma} \mu \geq \gamma \int_{\Gamma} \mu-k \int_{\Gamma} \mu
$$

Therefore, we deduce (22d) if $k \leq \gamma$.

For the time being, we admit that

$$
\gamma=\frac{\left[\tau_{n}(u, \hat{p})+\alpha_{n}, u_{n}+g_{n}\right]}{\beta},
$$

When $u_{n}+g_{n}=0$ on $\Gamma$, we have $\gamma=0$, but this is impossible because $u_{n}+g_{n} \geq 0$ and $\int_{\Gamma} g_{n} d \Gamma>0$. Therefore, we have by (35)

$$
\left[\tau_{n}(u, \hat{p})+\alpha_{n}, u_{n}+g_{n}\right]=\gamma \beta=\gamma \int_{\Gamma} g_{n} d \Gamma=\gamma \int_{\Gamma}\left(u_{n}+g_{n}\right) d \Gamma .
$$

Hence, taking

$$
k=\gamma,
$$

we obtain

$$
\left[\tau_{n}\left(u, p_{k}\right)+\alpha_{n}, u_{n}+g_{n}\right]=\left[\tau_{n}(u, \hat{p})+\alpha_{n}, u_{n}+g_{n}\right]-\gamma \int_{\Gamma}\left(u_{n}+g_{n}\right)=0 .
$$

Therefore, we have verified (22e). 
To show (35), we use $w^{*} \in V$ defined as (27) again. From (33) with $k=0$,

$$
\left[\tau_{n}(u, \hat{p})+\alpha_{n}, v_{n}+w_{n}^{*}\right] \geq\left[\tau_{n}(u, \hat{p})+\alpha_{n}, u_{n}+w_{n}^{*}\right] \quad\left(v \in K^{\sigma}\right) .
$$

Since $w^{*}=g$ on $\Gamma$, this is expressed equivalently as

$$
\left[\tau_{n}(u, \hat{p})+\alpha_{n}, v_{n}+g_{n}\right] \geq\left[\tau_{n}(u, \hat{p})+\alpha_{n}, u_{n}+g_{n}\right] \quad\left(v \in K^{\sigma}\right) .
$$

Moreover, we obtain

$$
\left[\tau_{n}(u, \hat{p})+\alpha_{n}, \frac{v_{n}+g_{n}}{\beta}\right] \geq\left[\tau_{n}(u, \hat{p})+\alpha_{n}, \frac{u_{n}+g_{n}}{\beta}\right] \quad\left(v \in K^{\sigma}\right) .
$$

Now let $\mu \in Y$ be arbitrary and set $\tilde{\mu}=\beta \mu-g_{n} \in M$. Since $\int_{\Gamma} \tilde{\mu} d \Gamma=0$, there exists $\tilde{v} \in V^{\sigma}$ such that $\tilde{v}_{n}=\tilde{\mu}$ on $\Gamma$ according to Remark 3 and [17, Lemma I.2.2]. Then, the function $\tilde{v}$ satisfies that $\tilde{v}_{n}+g_{n}=\beta \mu \geq 0$ on $\Gamma$. Consequently, $\tilde{v} \in K^{\sigma}$. Therefore, we have by (36) that

$$
\begin{aligned}
{\left[\tau_{n}(u, \hat{p})+\alpha_{n}, \mu\right] } & =\left[\tau_{n}(u, \hat{p})+\alpha_{n}, \frac{\tilde{\mu}+g_{n}}{\beta}\right] \\
& =\left[\tau_{n}(u, \hat{p})+\alpha_{n}, \frac{\tilde{v}_{n}+g_{n}}{\beta}\right] \geq \frac{1}{\beta}\left[\tau_{n}(u, \hat{p})+\alpha_{n}, u_{n}+g_{n}\right],
\end{aligned}
$$

which implies (35).

It remains to derive (24). First, from (30), we have

$$
\|\hat{p}\| \leq C \sup _{v \in V_{0}} \frac{|(f, v)-a(u, v)|}{\|v\|_{1}} \leq C(\|f\|+\|u\|),
$$

where we have used the standard infsup (Babuška and Brezzi's) condition (see [17, Corollary I.2.4])

$$
\inf _{q \in Q_{0}} \sup _{v \in V_{0}} \frac{b(q, v)}{\|q\|\|v\|_{1}} \geq C .
$$

Equation (22e), together with (20), implies

$$
a(u, u+g)+b(p, u+g)-(f, u+g)+\left[\alpha_{n}, u_{n}+g_{n}\right]=0 .
$$

Therefore, by virtue of Korn's inequality (see [24, Lemma 6.2]),

$$
C\|u+g\|_{1}^{2} \leq C\left(\|f\|+\|\alpha\|_{\left(M^{d}\right)^{\prime}}+\|g\|_{1}\right)\|u+g\|_{1}
$$

and, consequently,

$$
\|u+g\|_{1} \leq C_{*}, \quad\|u\|_{1} \leq C_{*} .
$$

Finally, because of the expression (35), we can estimate as

$$
|\gamma| \leq \frac{1}{\beta}\left\|\tau_{n}(u, \hat{p})+\alpha_{n}\right\|_{M^{\prime}}\left\|u_{n}+g_{n}\right\|_{\frac{1}{2}, \Gamma} \leq C\left(\|u\|_{1}+\|\hat{p}\|\right)\|u+g\|_{1} .
$$

Combining this with (37) and (38), we obtain (24).

\section{Finite element approximation}

While there are a lot of strategies for solving the variational inequality problem (VI), we concentrate our attention to its penalty approximation.

As a regularization of $[s]_{-}(s \in \mathbb{R})$, we introduce a function $\phi_{\delta}: \mathbb{R} \rightarrow \mathbb{R}$ that satisfies

$$
\begin{gathered}
\phi_{\delta} \text { is a non-increasing } C^{1}(\mathbb{R}) \text { function; } \\
\qquad \begin{array}{c}
\left|\phi_{\delta}(s)-[s]_{-}\right| \leq C \delta \quad(s \in \mathbb{R}) ; \\
\phi_{\delta}(s)=0 \quad(s \geq 0), \quad 0 \leq \phi_{\delta}(s) \leq-s \quad(s<0) ; \\
\left|\frac{d}{d s} \phi_{\delta}(s)\right| \leq C \quad(s \in \mathbb{R}),
\end{array}
\end{gathered}
$$


where $\delta \in(0,1]$ is a regularized parameter and $C$ 's are independent of $\delta$. As described in the Introduction, we can take, for example, the function $\phi_{\delta}(s)$ defined as $(3)$.

For penalty parameter $\varepsilon \in(0,1]$, we consider the following penalty problem,

$\left(\mathbf{P E} \mathbf{E}_{\varepsilon, \delta}\right)$ Find $(u, p) \in V \times Q$ such that

$$
\begin{aligned}
& a(u, v)+b(p, v)-\frac{1}{\varepsilon} \int_{\Gamma} \phi_{\delta}\left(u_{n}+g_{n}\right) v_{n} d \Gamma=(f, v)-[[\alpha, v]] \quad(\forall v \in V), \\
& b(q, u)=0 \\
& (\forall q \in Q) \text {. }
\end{aligned}
$$

This and the subsequent sections are devoted to the finite element approximation of $\left(\mathrm{PE}_{\varepsilon, \delta}\right)$. To avoid unimportant difficulties related to the "curved boundary", we consider only the case (G2). Consequently, the unit outer normal vector $n$ to $\Gamma$ is a constant vector over $\Gamma$.

We use the so-called MINI (P1b/P1) elements for discretization. Let $\left\{\mathcal{T}_{h}\right\}_{h}$ be a regular family of triangulations of $\Omega$. As the granularity parameter, we used $h=\max \left\{h_{T} \mid T \in \mathcal{T}_{h}\right\}$, where $h_{T}$ denotes the diameter of $T$. We introduce the following function spaces:

$$
\begin{gathered}
V_{h}=\left\{v_{h} \in C^{0}(\bar{\Omega}) \mid v_{h}=0 \text { on } S,\left.v_{h}\right|_{T} \in\left[\mathcal{P}_{1}^{(d)} \oplus \operatorname{span}\left\{\varphi_{T}\right\}\right]^{d}\left(\forall T \in \mathcal{T}_{h}\right)\right\}, \\
V_{0 h}=V_{h} \cap H_{0}^{1}(\Omega)^{d}, \quad V_{h}^{\sigma}=\left\{v_{h} \in V_{h} \mid b\left(q_{h}, v_{h}\right)=0\left(\forall q_{h} \in Q_{h}\right)\right\}, \\
Q_{h}=\left\{q_{h} \in C^{0}(\bar{\Omega})\left|q_{h}\right|_{T} \in \mathcal{P}_{1}^{(d)}\left(\forall T \in \mathcal{T}_{h}\right)\right\}, \quad Q_{0 h}=Q_{h} \cap Q_{0}, \\
M_{h}=\left\{\mu_{h}=\left.v_{h n}\right|_{\Gamma} \mid v_{h} \in V_{h}\right\}, \quad M_{0 h}=\left\{\mu_{h} \in M_{h} \mid \int_{\Gamma} \mu_{h} d \Gamma=0\right\} .
\end{gathered}
$$

Therein, $\mathcal{P}_{k}^{(d)}$ denotes the set of all polynomials in $x_{1}, \ldots, x_{d}$ of degree $\leq k$, and $\varphi_{T}=\prod_{i=1}^{d+1} \lambda_{T, i}$, with $\lambda_{T, 1}, \ldots, \lambda_{T, d+1}$ the barycentric coordinates of $T$.

We denote by $\mathcal{S}_{h}$ the $d-1$ dimensional triangulation of $\Gamma$ inherited from $\mathcal{T}_{h}$. We have

$$
M_{h}=\left\{\mu_{h} \in C(\bar{\Gamma})\left|\mu_{h}\right|_{S} \in \mathcal{P}_{1}^{(d-1)}\left(\forall S \in \mathcal{S}_{h}\right),\left.\mu_{h}\right|_{\partial \Gamma}=0\right\} \quad \text { (algebraically) }
$$

Moreover, we introduce a projection operator $\Lambda: Q \rightarrow Q_{0}$ by

$$
\Lambda q=q-m(q) \quad \text { with } \quad m(q)=\frac{1}{|\Omega|} \int_{\Omega} q d x \quad(q \in Q) .
$$

It seems readily apparent that $\|\Lambda q\| \leq C\|q\|$ for $q \in Q$ and $\Lambda q_{h} \in Q_{0 h}$ for $q_{h} \in Q_{h}$.

Then, the finite element approximation for $\left(\mathrm{PE}_{\varepsilon, \delta}\right)$ reads as follows.

$\left(\mathbf{P E}_{\varepsilon, \delta, h}\right)$ Find $\left(u_{h}, p_{h}\right) \in V_{h} \times Q_{h}$ such that

$$
\begin{array}{ll}
a\left(u_{h}, v_{h}\right)+b\left(p_{h}, v_{h}\right)-\frac{1}{\varepsilon} \int_{\Gamma} \phi_{\delta}\left(u_{n}+g_{n}\right) v_{h n} d \Gamma=\left(f, v_{h}\right)-\left[\left[\alpha, v_{h}\right]\right] & \left(\forall v_{h} \in V_{h}\right), \\
b\left(q_{h}, u_{h}\right)=0 & \left(\forall q_{h} \in Q_{h}\right) .
\end{array}
$$

Before considering the well-posedness and error estimates, we recall here basic results on the finite element method.

Babuška-Brezzi condition. As is well-known, the Babuška-Bezzi condition is well-known to hold true in $V_{0 h} \times Q_{0 h}$. There is a constant $\gamma^{\prime}>0$, which is independent of $h$, such that

$$
\inf _{q_{h} \in Q_{0 h}} \sup _{v_{h} \in V_{0 h}} \frac{b\left(q_{h}, v_{h}\right)}{\left\|v_{h}\right\|_{1}\left\|q_{h}\right\|} \geq \gamma^{\prime} .
$$


Discrete lifting operators and discrete traction vectors. The following is a discrete analogue of Lemma 2.

Lemma 8 ([23, Lemma 2.1]). (i) There is a continuous linear operator $\mathcal{E}_{h}$ from $M_{h}^{d}$ to $V_{h}$ such that $\mathcal{E}_{h} \mu_{h}=\mu_{h}$ on $\Gamma$ and $\left\|\mathcal{E}_{h} \mu_{h}\right\|_{1} \leq C\left\|\mu_{h}\right\|_{\frac{1}{2}, \Gamma}$ for any $\mu_{h} \in M_{h}^{d}$, where $C$ is independent of $h$.

(ii) There is a continuous linear operator $\mathcal{E}_{n h}$ from $M_{h}$ to $V_{h}$ such that $\left(\mathcal{E}_{n h} \mu_{h}\right)_{n}=\mu_{h}$ and $\left(\mathcal{E}_{n h} \mu_{h}\right)_{T}=0$ on $\Gamma$ and $\left\|\mathcal{E}_{n h} \mu_{h}\right\|_{1} \leq C\left\|\mu_{h}\right\|_{\frac{1}{2}, \Gamma}$ for any $\mu_{h} \in M_{h}$, where $C$ is independent of $h$.

(iii) For $\mu_{h} \in M_{0 h}$, the above $w_{h}=\mathcal{E}_{n h} \mu_{h}$ can be chosen in such way that $w_{h} \in V_{h}^{\sigma}$.

As the continuous case, we define traction vectors $\tau\left(u_{h}, p_{h}\right) \in\left(M_{h}^{d}\right)^{\prime}, \tau_{T}\left(u_{h}\right) \in\left(M_{h}^{d}\right)^{\prime}$ and $\tau_{n}\left(u_{h}, p_{h}\right) \in$ $M_{h}^{\prime}$ for a solution $\left(u_{h}, p_{h}\right) \in V_{h}^{\sigma} \times Q_{h}$ of

$$
a\left(u_{h}, v_{h}\right)+b\left(p_{h}, v_{h}\right)=\left(f, v_{h}\right) \quad\left(v_{h} \in V_{0 h}\right)
$$

as follows:

$$
\begin{gathered}
{\left[\left[\tau\left(u_{h}, p_{h}\right), \mu_{h}\right]\right]=a\left(u_{h}, w_{h}\right)+b\left(p_{h}, w_{h}\right)-\left(f, w_{h}\right) \quad\left(\mu_{h} \in M_{h}^{d}, w_{h}=\mathcal{E}_{h} \mu_{h}\right) ;} \\
{\left[\tau_{T}\left(u_{h}\right), \mu_{h}\right]=a\left(u_{h}, w_{h}\right)+b\left(p_{h}, w_{h}\right)-\left(f, w_{h}\right) \quad\left(\mu_{h} \in M_{h}^{d} \text { with } \mu_{h n}=0, w_{h}=\mathcal{E}_{h} \mu_{h}\right) ;} \\
{\left[\tau_{n}\left(u_{h}, p_{h}\right), \mu_{h}\right]=a\left(u_{h}, w_{h}\right)+b\left(p_{h}, w_{h}\right)-\left(f, w_{h}\right) \quad\left(\mu_{h} \in M_{h}, w_{h}=\mathcal{E}_{n h} \mu_{h}\right)}
\end{gathered}
$$

These definitions are independent of the way of extensions. In fact, for any $\mu_{h}$, let $w_{h} \in V_{h}$ and $\tilde{w}_{h} \in V_{h}$ be both extension of $\lambda_{h} ; w_{h n}=\tilde{w}_{h n}=\lambda_{h}$ on $\Gamma$. Set $v_{h}=w_{h}-\tilde{w}_{h}$. Then, since $v_{h} \in V_{0 h}$, we deduce, by $(46)$

$$
\begin{array}{r}
a\left(u_{h}, w_{h}\right)+b\left(p_{h}, w_{h}\right)-\left(f, w_{h}\right)-\left[a\left(u_{h}, \tilde{w}_{h}\right)+b\left(p_{h}, \tilde{w}_{h}\right)-\left(f, \tilde{w}_{h}\right)\right] \\
=a\left(u_{h}, v_{h}\right)+b\left(p_{h}, v_{h}\right)-\left(f, v_{h}\right)=0 .
\end{array}
$$

Consequently, (47c) is well-defined.

\section{Well-posedness of $\left(\mathrm{PE}_{\varepsilon, \delta, h}\right)$}

In this section, we establish the well-posedness of $\left(\mathrm{PE}_{\varepsilon, \delta, h}\right)$. Thus, we shall prove the following two theorems. Recall that $C_{*}$ denotes a positive constant depending only on $\Omega,\|f\|,\|g\|_{1}$ and $\|\alpha\|_{\left(M^{d}\right)^{\prime}}$.

Theorem 9. There exists a unique solution $\left(u_{h}, p_{h}\right) \in V_{h} \times Q_{h}$ of $\left(\mathrm{PE}_{\varepsilon, \delta, h}\right)$, and we have

$$
\left\|u_{h}\right\|_{1}+\left\|\hat{p}_{h}\right\|+\left\|\frac{1}{\varepsilon} \phi_{\delta}\left(u_{h n}+g_{n}\right)+k_{h}\right\|_{M_{h}^{\prime}} \leq C_{*},
$$

where $\hat{p}_{h}=\Lambda p_{h}$ and $k_{h}=m\left(p_{h}\right)$.

Theorem 10. Assume

(A1) the family $\left\{\mathcal{S}_{h}\right\}_{h}$ is quasi-uniform;

(A2) there exists $\Gamma_{1} \subset \Gamma$ with $\left|\Gamma_{1}\right|>0$ which is independent of $h, \varepsilon, \delta$ and $\Omega$ such that $u_{h n}+g_{n}>0$ on $\Gamma_{1}$.

Then, the solution $\left(u_{h}, p_{h}\right) \in V_{h} \times Q_{h}$ of $\left(\mathrm{PE}_{\varepsilon, \delta, h}\right)$ admits the following estimates:

$$
\begin{gathered}
\left\|u_{h}\right\|_{1}+\left\|p_{h}\right\|+\left\|\frac{1}{\varepsilon} \phi_{\delta}\left(u_{h n}+g_{n}\right)\right\|_{M_{h}^{\prime}} \leq C_{*} ; \\
\left\|\frac{1}{\varepsilon} \phi_{\delta}\left(u_{h n}+g_{n}\right)\right\|_{M^{\prime}} \leq C_{*}\left(1+\frac{h}{\varepsilon}\right) ; \\
\frac{1}{\sqrt{\varepsilon}}\left\|\left[u_{h n}+g_{n}\right]_{-}\right\|_{\Gamma} \leq C_{*}\left(1+\frac{\delta}{\varepsilon}\right) .
\end{gathered}
$$


Remark 11. Condition (A2) is not restrictive. It is natural to presume this condition if $\beta$ is sufficiently large and $h, \varepsilon, \delta$ are suitably small.

Remark 12. If $\delta \leq c_{0} \varepsilon$ with some $c_{0}>0$, we have, from (49c), $\left\|\left[u_{h n}+g_{n}\right]_{-}\right\|_{\Gamma} \rightarrow 0$ as $\varepsilon \rightarrow 0$.

To prove Theorem 9 , we apply the following fundamental result.

Lemma 13 ([27, Theorem 2.1]). Let $X$ be a separable reflexive Banach space and let $T: X \rightarrow X^{\prime}$ be a (possibly nonlinear) operator satisfying the following conditions:

1. (boundness) There exist $C, C^{\prime}, m>0$ s.t. $\|T u\|_{X^{\prime}} \leq C\|u\|_{X}^{m}+C^{\prime}$ for all $u \in X$;

2. (monotonicity) $\langle T u-T v, u-v\rangle_{X^{\prime}, X} \geq 0$ for all $u, v \in X$;

3. (hemicontinuity) For any $u, v, w \in X$, the function $\lambda \mapsto\langle T(u+\lambda v), w\rangle_{X^{\prime}, X}$ is continuous on $\mathbb{R}$;

4. (coerciveness) $\frac{\langle T u, u\rangle_{X^{\prime}, X}}{\|u\|_{X}} \rightarrow \infty$ as $\|u\|_{X} \rightarrow \infty$.

Then, for any $\varphi \in X^{\prime}$, there exists $u \in X$ such that $T u=\varphi$. Furthermore, if $T$ is strictly monotone:

$$
\langle T u-T v, u-v\rangle_{X^{\prime}, X}>0 \quad(\forall u, v \in X, u \neq v),
$$

then the solution is unique.

We set $\rho_{\delta}: V \rightarrow V^{\prime}$ by

$$
\left\langle\rho_{\delta}(u), v\right\rangle=-\int_{\Gamma} \phi_{\delta}\left(u_{n}+g_{n}\right) v_{n} d \Gamma \quad(v \in V) .
$$

Lemma 14. $\rho_{\delta}$ is a bounded, monotone and hemicontinuous operator from $V$ to $V^{\prime}$.

Proof. (boundness) By using (40c) and the trace theorem, we have

$$
\left\langle\rho_{\delta}(u), v\right\rangle \leq \int_{\Gamma}\left|u_{n}+g_{n}\right| \cdot\left|v_{n}\right| d \Gamma \leq\left(\|u\|_{1}+\left\|g_{n}\right\|_{\Gamma}\right)\|v\|_{1}
$$

for $u, v \in V$. Hence,

$$
\left\|\rho_{\delta}(u)\right\|_{V^{\prime}} \leq\|u\|_{1}+\left\|g_{n}\right\|_{\Gamma} \quad(u \in V) .
$$

(monotonicity) Since $-\phi_{\delta}(s)$ is non-decreasing function, we have

$$
\left\langle\rho_{\delta}(u)-\rho_{\delta}(v), u-v\right\rangle=-\int_{\Gamma}\left(\phi_{\delta}\left(u_{n}+g_{n}\right)-\phi_{\delta}\left(v_{n}+g_{n}\right)\right)\left(u_{n}+g_{n}-\left(v_{n}+g_{n}\right)\right) d \Gamma \geq 0
$$

for $u, v \in V$.

(hemicontinuity) Let $u, v, w \in V$. Then, a real-valued function

$$
\left\langle\rho_{\delta}(u+\lambda v), w\right\rangle=-\int_{\Gamma} \phi_{\delta}\left(u_{n}+\lambda v_{n}\right) w_{n} d \Gamma
$$

of $\lambda \in \mathbb{R}$ is a continuous function, since the function $\phi_{\delta}$ is continuous.

Proof of Theorem 9. It is divided into three steps.

Step 1. First, we prove that there exists a unique $u_{h} \in V_{h}^{\sigma}$ satisfying

$$
a\left(u_{h}, v_{h}\right)+\frac{1}{\varepsilon}\left\langle\rho_{\delta}\left(u_{h}\right), v_{h}\right\rangle=\left(f, v_{h}\right)-\left[\left[\alpha, v_{h}\right]\right] \quad\left(\forall v_{h} \in V_{h}^{\sigma}\right)
$$

by using Lemma 13 .

To do this, we introduce a nonlinear operator $A_{\varepsilon}: V_{h} \rightarrow V_{h}^{\prime}$ by setting

$$
\left\langle A_{\varepsilon} u_{h}, v_{h}\right\rangle=a\left(u_{h}, v_{h}\right)+\frac{1}{\varepsilon}\left\langle\rho_{\delta}\left(u_{h}\right), v_{h}\right\rangle \quad\left(u_{h}, v_{h} \in V_{h}\right) .
$$

and verify the conditions of Lemma 13. 
(boundness) For $u_{h} \in V_{h}^{\sigma}$, we have immediately

$$
\left\|A_{\varepsilon} u_{h}\right\|_{\left(V_{h}^{\sigma}\right)^{\prime}} \leq\left(\|a\|+\frac{1}{\varepsilon}\right)\left\|u_{h}\right\|_{1}+\frac{1}{\varepsilon}\left\|g_{n}\right\|_{\Gamma} .
$$

(strictly monotonicity) By virtue of Korn's inequality and monotonicity of $\rho_{\delta}$,

$$
\begin{aligned}
\left\langle A_{\varepsilon} u_{h}-A_{\varepsilon} v_{h}, u_{h}-v_{h}\right\rangle & =a\left(u_{h}-v_{h}, u_{h}-v_{h}\right)+\frac{1}{\varepsilon}\left\langle\rho_{\delta}\left(u_{h}\right)-\rho_{\delta}\left(v_{h}\right), u_{h}-v_{h}\right\rangle \\
& \geq C\left\|u_{h}-v_{h}\right\|_{1}^{2}>0
\end{aligned}
$$

for $u_{h}, v_{h} \in V_{h}^{\sigma}, u_{h} \neq v_{h}$.

(hemicontinuity) Let $u_{h}, v_{h}, w_{h} \in V_{h}^{\sigma}$. Then, a real-valued function

$$
\left\langle A_{\varepsilon}\left(u_{h}+\lambda v_{h}\right), w_{h}\right\rangle=a\left(u_{h}+\lambda v_{h}, w_{h}\right)+\frac{1}{\varepsilon}\left\langle\rho_{\delta}\left(u_{h}+\lambda v_{h}\right), w_{h}\right\rangle
$$

of $\lambda \in \mathbb{R}$ is continuous, since $a\left(\cdot, w_{h}\right)$ is continuous and $\rho_{\delta}(\cdot)$ is hemicontinuous. (coerciveness) For $u_{h} \in V_{h}^{\sigma}$, we have by (40c)

$$
\begin{aligned}
\left\langle\rho_{\delta}\left(u_{h}\right), u_{h}\right\rangle & =-\int_{\Gamma} \phi_{\delta}\left(u_{h n}+g_{n}\right) u_{h n} d \Gamma \\
& =-\int_{\Gamma} \phi_{\delta}\left(u_{h n}+g_{n}\right)\left(\left[u_{h n}+g_{n}\right]_{+}-\left[u_{h n}+g_{n}\right]_{-}-\left[g_{n}\right]_{+}+\left[g_{n}\right]_{-}\right) d \Gamma \\
& \geq-\int_{\Gamma} \phi_{\delta}\left(u_{n}+g_{n}\right)\left[g_{n}\right]_{-} d \Gamma \\
& \geq-C\left(\left\|u_{h}\right\|_{1}+\left\|g_{n}\right\|_{\Gamma}\right)\left\|g_{n}\right\|_{\Gamma} .
\end{aligned}
$$

This gives

$$
\frac{\left\langle A_{\varepsilon} u_{h}, u_{h}\right\rangle}{\left\|u_{h}\right\|_{1}}=\frac{a\left(u_{h}, u_{h}\right)}{\left\|u_{h}\right\|_{1}}+\frac{1}{\varepsilon} \frac{\left\langle\rho_{\delta}\left(u_{h}\right), u_{h}\right\rangle}{\left\|u_{h}\right\|_{1}} \geq C\left\|u_{h}\right\|_{1}-\frac{C}{\varepsilon} \frac{\left(\left\|u_{h}\right\|_{1}+\left\|g_{n}\right\|_{\Gamma}\right)}{\left\|u_{h}\right\|_{1}}\left\|g_{n}\right\|_{\Gamma},
$$

and, hence,

$$
\frac{\left\langle A_{\varepsilon} u_{h}, u_{h}\right\rangle}{\left\|u_{h}\right\|_{1}} \rightarrow \infty \quad \text { as } \quad\left\|u_{h}\right\|_{1} \rightarrow \infty
$$

As a consequence, we can apply Lemma 13 to conclude that there exists a unique $u_{h} \in V_{h}^{\sigma}$ satisfying $A_{\varepsilon} u_{h}=F_{h}$, where $F_{h} \in\left(V_{h}^{\sigma}\right)^{\prime}$ is defined as $\left\langle F, v_{h}\right\rangle=\left(f, v_{h}\right)-\left[\left[\alpha, v_{h}\right]\right]$ for $v_{h} \in V_{h}^{\sigma}$. Therefore, we have proved a unique existence of the solution $u_{h} \in V_{h}^{\sigma}$ of (50).

Step 2. We verify the unique existence of $p_{h} \in Q_{h}$ such that $\left(u_{h}, p_{h}\right)$ is a solution of $\left(\mathrm{PE}_{\varepsilon, \delta, h}\right)$. In view of (45), there exists a unique $\hat{p}_{h} \in Q_{0 h}$ satisfying

$$
a\left(u_{h}, v_{h}\right)+b\left(\hat{p}_{h}, v_{h}\right)=\left(f, v_{h}\right) \quad\left(v_{h} \in V_{0 h}\right) .
$$

Now, we find a constant $k_{h}$ such that $\left(u_{h}, p_{h}\right)$ is a solution of $\left(\mathrm{PE}_{\varepsilon, \delta, h}\right)$, where $p_{h}=\hat{p}_{h}+k_{h}$. (In fact, with any $k_{h} \in \mathbb{R},\left(u_{h}, p_{h}\right)$ also solves (46).) To do this, we first rewrite (44a) as by using (47a)-(47c),

$$
\left[\tau_{n}\left(u_{h}, p_{h}\right)-\varepsilon^{-1} \phi_{\delta}\left(u_{h n}+g_{n}\right)-\alpha_{n}, v_{h n}\right]+\left[\left[\tau_{T}\left(u_{h}\right)-\alpha_{T}, v_{h T}\right]\right]=0 \quad\left(v_{h} \in V_{h}\right) .
$$

Consequently, in view of Lemma 8, it suffices to prove the following two equations:

$$
\begin{gathered}
{\left[\tau_{n}\left(u_{h}, p_{h}\right)-\varepsilon^{-1} \phi_{\delta}\left(u_{h n}+g_{n}\right)-\alpha_{n}, \mu_{h}\right]=0 \quad\left(\mu_{h} \in M_{h}\right) ;} \\
{\left[\left[\tau_{T}\left(u_{h}\right)-\alpha_{T}, \mu_{h}\right]\right]=0 \quad\left(\mu_{h} \in M_{h}^{d} \text { with } \mu_{h n}=0\right) .}
\end{gathered}
$$

It might be readily apparent that $v_{h}=\mathcal{E}_{h} \mu_{h} \in V_{h}$ belongs to $V_{h}^{\sigma}$ for any $\mu_{h} \in M_{h}^{d}$ with $\mu_{h n}=0$. Hence, (50) and (47b) immediately implies (52b). 
On the other hand, combining (50) and (47c), we have

$$
\left[\tau_{n}\left(u_{h}, p_{h}\right)+\alpha_{n}-\varepsilon^{-1} \phi_{\delta}\left(u_{h n}+g_{n}\right), \lambda_{h}\right]=0 \quad\left(\forall \lambda_{h} \in M_{0 h}\right) .
$$

At this stage, let us take

$$
\tilde{\mu}_{h} \in Y_{h}=\left\{\mu_{h} \in M_{h} \mid \mu_{h} \geq 0, \mu_{h} \not \equiv 0, \int_{\Gamma} \mu_{h} d \Gamma=1\right\} .
$$

Then, for any $\mu_{h} \in M_{h}$, the function $\mu_{h}-\kappa_{h} \tilde{\mu}_{h}$ belongs to $M_{0 h}$, where $\kappa_{h}=\int_{\Gamma} \mu_{h} d \Gamma$.

Therefore, for any $\mu_{h} \in M_{h}$,

$$
\begin{aligned}
{\left[\tau_{n}\left(u_{h}, p_{h}\right)+\alpha_{n}-\varepsilon^{-1} \phi_{\delta}\left(u_{h n}+g_{n}\right), \mu_{h}\right]=} & {\left[\tau_{n}\left(u_{h}, p_{h}\right)+\alpha_{n}-\varepsilon^{-1} \phi_{\delta}\left(u_{h n}+g_{n}\right), \mu_{h}-\kappa_{h} \tilde{\mu}_{h}\right] } \\
& +\left[\tau_{n}\left(u_{h}, p_{h}\right)+\alpha_{n}-\varepsilon^{-1} \phi_{\delta}\left(u_{h n}+g_{n}\right), \kappa_{h} \tilde{\mu}_{h}\right] \\
= & \kappa_{h}\left[\tau_{n}\left(u_{h}, p_{h}\right)+\alpha_{n}-\varepsilon^{-1} \phi_{\delta}\left(u_{h n}+g_{n}\right), \tilde{\mu}_{h}\right]
\end{aligned}
$$

Now, choosing

$$
k_{h}=\left[\tau_{n}\left(u_{h}, \hat{p}_{h}\right)-\varepsilon^{-1} \phi_{\delta}\left(u_{h n}+g_{n}\right), \tilde{\mu}_{h}\right]
$$

we have

$$
\begin{aligned}
{\left[\tau_{n}\left(u_{h}, p_{h}\right)+\alpha_{n}-\varepsilon^{-1} \phi_{\delta}\left(u_{h n}+g_{n}\right), \tilde{\mu}_{h}\right] } & =\left[\tau_{n}\left(u_{h}, \hat{p}_{h}\right)+\alpha_{n}-\varepsilon^{-1} \phi_{\delta}\left(u_{h n}+g_{n}\right), \tilde{\mu}_{h}\right]-k_{h} \\
& =0 .
\end{aligned}
$$

Hence, we get (52a) by (54).

It remains to be verified that (55) is independent of the choice of $\tilde{\mu}_{h}$. We let $\tilde{\mu}_{h}, \tilde{\mu}_{h}^{\prime} \in Y_{h}$ with $\tilde{\mu}_{h} \neq \tilde{\mu}_{h}^{\prime}$ and let the corresponding $k_{h}$ be denoted by $\tilde{k}_{h}, \tilde{k}_{h}^{\prime}$, respectively. Then, since $\lambda_{h}=\mu_{h}-\mu_{h}^{\prime}$ satisfies $\int_{\Gamma} \lambda_{h} d \Gamma=0$, we have by $(53)$,

$$
\tilde{k}_{h}-\tilde{k}_{h}^{\prime}=\left[\tau_{n}\left(u_{h}, \hat{p}_{h}\right)+\alpha_{n}-\varepsilon^{-1} \phi_{\delta}\left(u_{h n}+g_{n}\right), \lambda_{h}\right]=0,
$$

which means that $k_{h}$ is uniquely determined by (55).

Step 3. Finally, we derive the stability result (48). Substituting $v_{h}=u_{h} \in V_{h}^{\sigma}$ into (50), we obtain,

$$
a\left(u_{h}, u_{h}\right)-\frac{1}{\varepsilon} \int_{\Gamma} \phi_{\delta}\left(u_{h n}+g_{n}\right) u_{h n} d \Gamma=\left(f, u_{h}\right)-\left[\left[\alpha, u_{h}\right]\right] .
$$

Noting that, by (40c)

$$
\begin{aligned}
-\frac{1}{\varepsilon} \int_{\Gamma} \phi_{\delta}\left(u_{h n}+g_{n}\right) u_{h n} d \Gamma & =-\frac{1}{\varepsilon} \int_{\Gamma} \phi_{\delta}\left(u_{h n}+g_{n}\right)\left(u_{h n}+g_{n}\right) d \Gamma+\frac{1}{\varepsilon} \int_{\Gamma} \phi_{\delta}\left(u_{h n}+g_{n}\right) g_{n} d \Gamma \\
& \geq \frac{1}{\varepsilon} \int_{\Gamma} \phi_{\delta}\left(u_{h n}+g_{n}\right)\left[u_{h n}+g_{n}\right]_{-} d \Gamma \geq 0,
\end{aligned}
$$

we get

$$
a\left(u_{h}, u_{h}\right) \leq\left(f, u_{h}\right)-\left[\left[\alpha, u_{h}\right]\right] .
$$

Hence, by virtue of Korn's inequality ([24, Lemma 6.2]),

$$
\left\|u_{h}\right\|_{1} \leq C\left(\|f\|+\|\alpha\|_{\left(M^{d}\right)^{\prime}}\right) .
$$

Moreover, according to (45) and (51),

$$
\left\|\hat{p}_{h}\right\| \leq \sup _{v_{h} \in V_{0 h}} \frac{b\left(\hat{p}_{h}, v_{h}\right)}{\left\|v_{h}\right\|_{1}}=\sup _{v_{h} \in V_{0 h}} \frac{\left(f, v_{h}\right)-a\left(u_{h}, v_{h}\right)}{\left\|v_{h}\right\|_{1}} \leq C\left(\|f\|+\left\|u_{h}\right\|_{1}\right) .
$$

Since (44a) is expressed as

$$
\begin{aligned}
\int_{\Gamma}\left(\varepsilon^{-1} \phi_{\delta}\left(u_{h n}+g_{n}\right)+\right. & \left.k_{h}\right) \mu_{h} d \Gamma \\
& =a\left(u_{h}, v_{h}\right)+b\left(\hat{p}_{h}, v_{h}\right)-\left(f, v_{h}\right)+\left[\left[\alpha, v_{h}\right]\right] \quad\left(\forall \mu_{h} \in M_{h}, v_{h}=\mathcal{E}_{n h} \mu_{h} \in V_{h}\right)
\end{aligned}
$$


we deduce

$$
\left\|\frac{1}{\varepsilon} \phi_{\delta}\left(u_{h n}+g_{n}\right)+k_{h}\right\|_{M_{h}^{\prime}} \leq C\left(\left\|u_{h}\right\|_{1}+\left\|\hat{p}_{h}\right\|+\|f\|+\|\alpha\|_{\left(M^{d}\right)^{\prime}}\right) .
$$

Summing up (58), (59) and (60), we obtain (48).

We proceed to the proof of Theorem 10. We use the standard Lagrange interpolation operator $i_{h}$ : $C(\bar{\Gamma}) \rightarrow M_{h}$ defined by

$$
i_{h} \mu(P)=\mu(P) \quad\left(\text { every node } P \text { of } \mathcal{S}_{h}\right)
$$

and the $L^{2}$ projection operator $\pi_{h}: L^{2}(\Gamma) \rightarrow M_{h}$ defined by

$$
\int_{\Gamma}\left(\pi_{h} \mu-\mu\right) \mu_{h} d \Gamma=0 \quad\left(\mu_{h} \in M_{h}\right)
$$

The following results are well-known.

$$
\begin{gathered}
\mu \geq 0 \quad \Rightarrow \quad i_{h} \mu \geq 0, \\
\left\|i_{h} \mu-\mu\right\|_{\Gamma}+h\left\|i_{h} \mu-\mu\right\|_{1, \Gamma} \leq C h^{2}|\mu|_{2, \Gamma} \quad\left(\mu \in H^{2}(\Gamma) \cap H_{0}^{1}(\Gamma)\right), \\
\left\|\pi_{h} \mu\right\|_{\Gamma} \leq C\|\mu\|_{\Gamma} \quad\left(\mu \in L^{2}(\Gamma)\right), \\
\left\|\pi_{h} \mu\right\|_{1, \Gamma} \leq C\|\mu\|_{1, \Gamma} \quad\left(\mu \in H_{0}^{1}(\Gamma)\right), \\
\left\|\pi_{h} \mu-\mu\right\|_{\Gamma} \leq C h\|\mu\|_{1, \Gamma} \quad\left(\mu \in H_{0}^{1}(\Gamma)\right) .
\end{gathered}
$$

In fact, $(63 \mathrm{a}),(63 \mathrm{~b}),(63 \mathrm{c}),(63 \mathrm{e})$ are standard. On the other hand, (63d) holds true if $\left\{\mathcal{S}_{h}\right\}_{h}$ is quasiuniform (see $[22,14,6]$ ).

Remark 15. According to $(63 \mathrm{~b}),\left\|i_{h} \mu\right\|_{1, \Gamma}$ is bounded by a positive constant depending only on $\mu$ if $\mu \in C_{0}^{\infty}(\Gamma)$.

Lemma 16. $\left\|\pi_{h} \mu-\mu\right\|_{M^{\prime}} \leq C h\|\mu\|_{\frac{1}{2}, \Gamma}$ for any $\mu \in M$.

Proof. It follows from (63c) that $\left\|\pi_{h} \mu-\mu\right\|_{\Gamma} \leq C\|\mu\|_{\Gamma}$. Combining this with (63e), (15) and applying the interpolation theorem (see[29, Theorem 5.1, Ch. 1]), we obtain

$$
\left\|\pi_{h} \mu-\mu\right\|_{\Gamma} \leq C h^{\frac{1}{2}}\|\mu\|_{\frac{1}{2}, \Gamma} \quad(\mu \in M) .
$$

We can use this in the following way. That is, noting (62),

$$
\begin{aligned}
\left\|\pi_{h} \mu-\mu\right\|_{M^{\prime}} & =\sup _{\lambda \in M} \frac{\left[\pi_{h} \mu-\mu, \lambda\right]}{\|\lambda\|_{\frac{1}{2}, \Gamma}}=\sup _{\lambda \in M} \frac{\left[\pi_{h} \mu-\mu, \pi_{h} \lambda-\lambda\right]}{\|\lambda\|_{\frac{1}{2}, \Gamma}} \\
& \leq \sup _{\lambda \in M} \frac{\left\|\pi_{h} \mu-\mu\right\|_{\Gamma}\left\|\pi_{h} \lambda-\lambda\right\|_{\Gamma}}{\|\lambda\|_{\frac{1}{2}, \Gamma}} \leq C h\|\mu\|_{\frac{1}{2}, \Gamma} .
\end{aligned}
$$

Lemma 17. $\left\|\phi_{\delta}(\mu)\right\|_{\frac{1}{2}, \Gamma} \leq C\|\mu\|_{\frac{1}{2}, \Gamma}$ for any $\mu \in M$.

Proof. Using (40c) and (40d), we have $\left\|\phi_{\delta}(\mu)\right\|_{\Gamma} \leq C\|\mu\|_{\Gamma}$ for $\mu \in Q$ and $\left\|\phi_{\delta}(\mu)\right\|_{1, \Gamma} \leq C\|\mu\|_{1, \Gamma}$ for $\mu \in H_{0}^{1}(\Gamma)$. Hence, we can apply the (nonlinear) interpolation theorem (see [28, Theorem 3.1]) and (15) to get the desired result.

Proof of Theorem 10. First, we derive an estimation for $k_{h}$. We take $\tilde{\mu} \in C_{0}^{\infty}(\Gamma)$ satisfying

$$
\tilde{\mu} \geq 0, \tilde{\mu} \not \equiv 0 \text { in } \Gamma, \quad \operatorname{supp} \tilde{\mu} \subset \Gamma_{1} .
$$

Then, setting $\tilde{\mu}_{h}=i_{h} \mu \in M_{h}$, we have

$$
\tilde{\mu}_{h} \geq 0, \tilde{\mu} \not \equiv 0 \text { in } \Gamma, \quad \tilde{\mu}_{h}=0 \text { in } \Gamma \backslash \Gamma_{1}, \quad\left\|\tilde{\mu}_{h}\right\|_{M} \leq C, \quad\left|\int_{\Gamma} \tilde{\mu}_{h} d \Gamma-\int_{\Gamma} \tilde{\mu} d \Gamma\right| \leq C h^{2},
$$


where those $C$ 's depend on $\mu$.

Since (A2) gives

we deduce from (44a) and (64)

$$
\phi_{\delta}\left(u_{h n}+g_{n}\right)=0 \quad \text { on } \Gamma_{1},
$$

$$
\begin{aligned}
k_{h} \int_{\Gamma} \tilde{\mu}_{h} d \Gamma & =a\left(u_{h}, \tilde{v}_{h}\right)+b\left(\hat{p}_{h}, \tilde{v}_{h}\right)-\frac{1}{\varepsilon} \int_{\Gamma} \phi_{\delta}\left(u_{h n}+g_{n}\right) \tilde{\mu}_{h} d \Gamma-\left(f, \tilde{v}_{h}\right)+\left[\left[\alpha, \tilde{v}_{h}\right]\right] \\
& =a\left(u_{h}, \tilde{v}_{h}\right)+b\left(\hat{p}_{h}, \tilde{v}_{h}\right)-\left(f, \tilde{v}_{h}\right)+\left[\left[\alpha, \tilde{v}_{h}\right]\right],
\end{aligned}
$$

where $\tilde{v}_{h}=\mathcal{E}_{n h} \tilde{\mu}_{h} \in V_{h}$

This leads to

$$
\left|k_{h}\right| \leq C_{*}, \quad\left\|p_{h}\right\| \leq C_{*} .
$$

Hence, we have proved (49a).

Using (62) and (44a), we can make the following calculation.

$$
\begin{aligned}
\int_{\Gamma} \varepsilon^{-1} \pi_{h} \phi_{\delta}\left(u_{h n}+g_{n}\right) \mu_{h} d \Gamma & =\int_{\Gamma} \varepsilon^{-1} \phi_{\delta}\left(u_{h n}+g_{n}\right) \mu_{h} d \Gamma \\
= & a\left(u_{h}, v_{h}\right)+b\left(p_{h}, v_{h}\right)-\left(f, v_{h}\right)+\left[\left[\alpha, v_{h}\right]\right] \quad\left(\forall \mu_{h} \in M_{h}, v_{h}=\mathcal{E}_{n h} \mu_{h} \in V_{h}\right) .
\end{aligned}
$$

Therefore,

$$
\left\|\varepsilon^{-1} \pi_{h} \phi_{\delta}\left(u_{h n}+g_{n}\right)\right\|_{M_{h}^{\prime}} \leq\left(\left\|u_{h}\right\|_{1}+\left\|\hat{p}_{h}\right\|+\|f\|+\|\alpha\|_{\left(M^{d}\right)^{\prime}}+\|g\|_{1}\right) .
$$

We write as

$$
\begin{aligned}
\sup _{\mu \in M} \frac{\left[\varepsilon^{-1} \phi_{\delta}\left(u_{h n}+g_{n}\right), \mu\right]}{\|\mu\|_{\frac{1}{2}, \Gamma}} & \\
= & \underbrace{\frac{1}{\varepsilon} \sup _{\mu \in M} \frac{\left[\phi_{\delta}\left(u_{h n}+g_{n}\right)-\pi_{h} \phi_{\delta}\left(u_{h n}+g_{n}\right), \mu\right]}{\|\mu\|_{\frac{1}{2}, \Gamma}}}_{=I_{1}}+\underbrace{\sup _{\mu \in M} \frac{\left[\varepsilon^{-1} \pi_{h} \phi_{\delta}\left(u_{h n}+g_{n}\right), \mu\right]}{\|\mu\|_{\frac{1}{2}, \Gamma}}}_{=I_{2}} .
\end{aligned}
$$

Using Lemmas 16 and 17,

$$
\begin{aligned}
\left\|\pi_{h} \phi_{\delta}\left(u_{h n}+g_{n}\right)-\phi_{\delta}\left(u_{h n}+g_{n}\right)\right\|_{M^{\prime}} & \leq C h\left\|\phi_{\delta}\left(u_{h n}+g_{n}\right)\right\|_{\frac{1}{2}, \Gamma} \\
& \leq C h\left\|u_{h n}+g_{n}\right\|_{\frac{1}{2}, \Gamma} \\
& \leq C h\left(\left\|u_{h}\right\|_{1}+\|g\|_{1}\right) .
\end{aligned}
$$

Consequently,

$$
\left|I_{1}\right| \leq C \frac{h}{\varepsilon}\left(\left\|u_{h}\right\|_{1}+\|g\|_{1}\right)
$$

On the other hand, by virtue of (62), (63d) and (67), we have

$$
\begin{aligned}
I_{2} & =\sup _{\mu \in M} \frac{\left[\varepsilon^{-1} \pi_{h} \phi_{\delta}\left(u_{h n}+g_{n}\right), \mu\right]}{\|\mu\|_{\frac{1}{2}, \Gamma}} \leq C \sup _{\mu \in M} \frac{\left[\varepsilon^{-1} \pi_{h} \phi_{\delta}\left(u_{h n}+g_{n}\right), \pi_{h}\right]}{\left\|\pi_{h} \mu\right\|_{\frac{1}{2}, \Gamma}} \\
& \leq C \sup _{\mu_{h} \in M_{h}} \frac{\left[\varepsilon^{-1} \pi_{h} \phi_{\delta}\left(u_{h n}+g_{n}\right), \mu_{h}\right]}{\left\|\mu_{h}\right\|_{\frac{1}{2}, \Gamma}}
\end{aligned}
$$

Therefore, from (67),

$$
\left|I_{2}\right| \leq C\left(\left\|u_{h}\right\|_{1}+\left\|p_{h}\right\|+\|f\|+\|\alpha\|_{\left(M^{d}\right)^{\prime}}+\|g\|_{1}\right)
$$

Summing up those estimates, we get (49b).

Finally, using (40b),

$$
\begin{aligned}
-\frac{1}{\varepsilon} \int_{\Gamma} \phi_{\delta}\left(u_{h n}+g_{n}\right) u_{h n} d \Gamma & \geq \frac{1}{\varepsilon} \int_{\Gamma} \phi_{\delta}\left(u_{h n}+g_{n}\right)\left[u_{h n}+g_{n}\right]_{-} d \Gamma \geq 0 \\
& \geq \frac{1}{\varepsilon} \int_{\Gamma}\left(\left[u_{h n}+g_{n}\right]_{-}^{2}-C \delta\left[u_{h n}+g_{n}\right]_{-}\right) d \Gamma \geq 0
\end{aligned}
$$


We apply this to (56) and obtain

$$
\begin{aligned}
\frac{1}{\varepsilon} \int_{\Gamma}\left[u_{h n}+g_{n}\right]_{-}^{2} d \Gamma & \leq\left(f, u_{h}\right)-\left[\left[\alpha, u_{h}\right]\right]+C \frac{\delta}{\varepsilon} \int_{\Gamma}\left[u_{h n}+g_{n}\right]_{-} d \Gamma \\
& \leq\|f\| \cdot\left\|u_{h}\right\|_{1}+\|\alpha\|_{\left(M^{d}\right)^{\prime}}\left\|u_{h}\right\|_{1}+C \frac{\delta}{\varepsilon}\left(\left\|u_{h}\right\|_{1}+\|g\|_{1}\right),
\end{aligned}
$$

which implies (49c).

\section{Error estimate}

We are now ready to state the error estimates between $(\mathrm{PDE})$ and $\left(\mathrm{PE}_{\varepsilon, \delta, h}\right)$.

Theorem 18. Assume that (A1) and (A2) are satisfied. Let $(u, p)$ and $\left(u_{h}, p_{h}\right)$ be solutions of (PDE) and $\left(\mathrm{PE}_{\varepsilon, \delta, h}\right)$, respectively, and suppose that $(u, p) \in H^{2}(\Omega)^{d} \times H^{1}(\Omega)$ and $\tau_{n}(u, p)+\alpha_{n} \in M$. Moreover, assume that $h, \varepsilon, \delta$ are sufficiently small and $h \leq c_{1} \varepsilon$ with a constant $c_{1}>0$, then we have

$$
\left\|u-u_{h}\right\|_{1}+\left\|p-p_{h}\right\| \leq C_{* *}\left(h+\varepsilon+\sqrt{\frac{\delta^{2}}{\varepsilon}}\right)
$$

and

$$
\begin{array}{r}
\left\|\tau_{n}(u, p)+\alpha_{n}-\frac{1}{\varepsilon} \phi_{\delta}\left(u_{h n}+g_{n}\right)\right\|_{M^{\prime}}+\sqrt{\varepsilon}\left\|\tau_{n}(u, p)+\alpha_{n}-\frac{1}{\varepsilon} \phi_{\delta}\left(u_{h n}+g_{n}\right)\right\|_{\Gamma} \\
\leq C_{* *}\left(h+\varepsilon+\sqrt{\frac{\delta^{2}}{\varepsilon}}\right),
\end{array}
$$

where $C_{* *}$ denotes a positive constant depending only on $c_{1}, \Omega,|u|_{2},|p|_{1},\left\|\tau_{n}(u, p)+\alpha_{n}\right\|_{M},\|f\|,\|g\|_{1}$ and $\|\alpha\|_{\left(M^{d}\right)^{\prime}}$. Particularly, if taking as $c_{2} \varepsilon \leq h$ and $\delta \leq c_{3} h^{\frac{3}{2}}$ with constants $\left(c_{1}>\right) c_{2}, c_{3}>0$, we have the optimal-order error estimate

$$
\left\|u-u_{h}\right\|_{1}+\left\|p-p_{h}\right\| \leq C_{* *} h .
$$

We use the standard Lagrange interpolation operator $I_{h}: C(\Omega)^{d} \rightarrow V_{h}$ and the $L^{2}$ projection operator $\Pi_{h}: Q \rightarrow Q_{h}$. Actually, the following are well known.

$$
\begin{gathered}
\left\|v-I_{h} v\right\|_{1} \leq C h|v|_{2} \quad\left(v \in\left[H^{2}(\Omega) \cap H_{0}^{1}(\Omega)\right]^{d}\right), \\
\left\|q-\Pi_{h} q\right\| \leq C h|q|_{1} \quad\left(q \in H^{1}(\Omega)\right) .
\end{gathered}
$$

Proof of Theorem 18. It is divided into three steps.

Step 1. Let us show that the following non-optimal error estimate holds true:

$$
\left\|u-u_{h}\right\|_{1}+\left\|\hat{p}-\hat{p}_{h}\right\| \leq C_{* *}(\sqrt{\varepsilon}+\sqrt{\delta}+\sqrt{h}),
$$

where $\hat{p}=\Lambda p, \hat{p}_{h}=\Lambda p_{h}$.

We recall that (22) together with (20) give

$$
a(u, v)+b(p, v)-\left[\tau_{n}(u, p), v_{n}\right]=(f, v)-\left[\left[\alpha_{T}, v_{T}\right]\right] \quad(v \in V) .
$$

Hence, errors $u-u_{h}$ and $p-p_{h}$ satisfy

$$
a\left(u-u_{h}, v_{h}\right)+b\left(p-p_{h}, v_{h}\right)-\left[\tau_{n}(u, p)+\alpha_{n}-\varepsilon^{-1} \phi_{\delta}\left(u_{h n}+g_{n}\right), v_{h n}\right]=0 \quad\left(v_{h} \in V_{h}\right) .
$$

Setting $\hat{p}=\Lambda p, \hat{p}_{h}=\Lambda p_{h}, k=m(p)$ and $k_{h}=m\left(p_{h}\right)$, we can write as

$$
\begin{aligned}
a\left(u-u_{h}, v_{h}\right)= & \underbrace{-b\left(\hat{p}-\hat{p}_{h}, v_{h}\right)}_{=J_{1}\left(v_{h}\right)} \\
& \underbrace{+\left[\tau_{n}(u, p)+\alpha_{n}-\varepsilon^{-1} \phi_{\delta}\left(u_{h n}+g_{n}\right)+k-k_{h}, v_{h n}\right]}_{=J_{2}\left(v_{h}\right)} \quad\left(v_{h} \in V_{h}\right) .
\end{aligned}
$$


Particularly we have

$$
b\left(\Lambda q_{h}-\hat{p}_{h}, v_{h}\right)=-a\left(u-u_{h}, v_{h}\right)-b\left(\hat{p}-\Lambda q_{h}, v_{h}\right) \quad\left(v_{h} \in V_{0 h}, q_{h} \in Q_{h}\right) .
$$

and, by application of (45),

$$
\begin{aligned}
\left\|\Lambda q_{h}-\hat{p}_{h}\right\| & \leq C \sup _{v_{h} \in V_{0 h}} \frac{-a\left(u-u_{h}, v_{h}\right)-b\left(\hat{p}-\Lambda q_{h}, v_{h}\right)}{\left\|v_{h}\right\|_{1}} \\
& \leq C\left(\left\|u-u_{h}\right\|_{1}+\left\|\hat{p}-\Lambda q_{h}\right\|\right) \\
& \leq C\left(\left\|u-u_{h}\right\|_{1}+\left\|p-q_{h}\right\|\right) \quad\left(q_{h} \in Q_{h}\right) .
\end{aligned}
$$

At this stage, we set

$$
v_{h}=I_{h} u-u_{h} \in V_{h}, \quad q_{h}=\Pi_{h} p \in Q_{h}, \quad \hat{q}_{h}=\Lambda q_{h} \in Q_{0 h} .
$$

Then,

$$
\begin{aligned}
\left\|\hat{p}-\hat{p}_{h}\right\| & \leq\left\|\hat{p}-\hat{q}_{h}\right\|+\left\|\hat{q}_{h}-\hat{p}_{h}\right\| \\
& \leq\left\|p-\Pi_{h} p\right\|+C\left(\left\|u-u_{h}\right\|_{1}+\left\|p-\Pi_{h} p\right\|\right) \\
& \leq C_{* *} h+C\left\|u-u_{h}\right\|_{1} .
\end{aligned}
$$

Using (70), (74) and $\left\|\hat{p}-\hat{q}_{h}\right\| \leq C\left\|p-q_{h}\right\|$, we estimate as

$$
\begin{aligned}
\left|J_{1}\left(I_{h} u-u_{h}\right)\right| & \leq\left|b\left(\hat{p}-\hat{p}_{h}, I_{h} u-u\right)\right|+\left|b\left(\hat{p}-\hat{q}_{h}, u-u_{h}\right)\right|+\left|b\left(\hat{q}-\hat{p}_{h}, u-u_{h}\right)\right| \\
& \leq\|b\| \cdot\left\|\hat{p}-\hat{p}_{h}\right\| \cdot\left\|I_{h} u-u\right\|_{1}+\|b\| \cdot\left\|\hat{p}-\hat{q}_{h}\right\| \cdot\left\|u-u_{h}\right\|_{1}+0 \\
& \leq C\left(C_{* *} h+\left\|u-u_{h}\right\|_{1}\right) \cdot h|u|_{2}+C h|p|_{1}\left\|u-u_{h}\right\|_{1} \\
& \leq C_{* *} h^{2}+C_{* *} h\left\|u-u_{h}\right\|_{1} \\
& \leq C_{* *} h^{2}+C_{* *} h\left\|I_{h} u-u_{h}\right\|_{1} .
\end{aligned}
$$

To perform an estimation for $J_{2}$, we divide it as

$$
\begin{aligned}
J_{2}\left(I_{h} u-u_{h}\right)=\underbrace{\left[\tau_{n}(u, p)+\alpha_{n}-\varepsilon^{-1} \phi_{\delta}\left(u_{h n}+g_{n}\right)+k-k_{h},\left(I_{h} u\right)_{n}-u_{n}\right]}_{=J_{21}} \\
\underbrace{+\left[\tau_{n}(u, p)+\alpha_{n}-\varepsilon^{-1} \phi_{\delta}\left(u_{h n}+g_{n}\right)+k-k_{h}, u_{n}-u_{h n}\right]}_{=J_{22}} .
\end{aligned}
$$

According to stability results (48) and (49b), we deduce

$$
\begin{aligned}
\left|J_{21}\right| & \leq\left(\left\|\tau_{n}(u, \hat{p})\right\|_{M^{\prime}}+\left\|\varepsilon^{-1} \phi_{\delta}\left(u_{h n}+g_{n}\right)\right\|_{M^{\prime}}+\left\|\alpha_{n}\right\|_{M^{\prime}}+\left|k_{h}\right|\right)\left\|\left(I_{h} u\right)_{n}-u_{n}\right\|_{\frac{1}{2}, \Gamma} \\
& \leq C_{*}\left\|I_{h} u-u\right\|_{1} \leq C_{* *} h .
\end{aligned}
$$

Noting

$$
\int_{\Gamma}\left(u_{n}-u_{h n}\right) d \Gamma=\int_{\Omega} \nabla \cdot\left(u-u_{h}\right) d x=0
$$


and using (22c), (22d), (22e), (40b), (40c) and (49b), we can calculate as:

$$
\begin{aligned}
& J_{22}=\left[\tau_{n}(u, p)+\alpha_{n}-\varepsilon^{-1} \phi_{\delta}\left(u_{h n}+g_{n}\right), u_{n}+g_{n}-\left(u_{h n}+g_{n}\right)\right] \\
& =-\left[\varepsilon^{-1} \phi_{\delta}\left(u_{h n}+g_{n}\right), u_{n}+g_{n}\right]-\left[\tau_{n}(u, p)+\alpha_{n}, u_{h n}+g_{n}\right] \\
& +\left[\varepsilon^{-1} \phi_{\delta}\left(u_{h n}+g_{n}\right), u_{h n}+g_{n}\right] \\
& =\underbrace{-\left[\varepsilon^{-1} \phi_{\delta}\left(u_{h n}+g_{n}\right), u_{n}+g_{n}\right]}_{\leq 0} \underbrace{-\left[\tau_{n}(u, p)+\alpha_{n},\left[u_{h n}+g_{n}\right]_{+}\right]}_{\leq 0} \\
& +\left[\tau_{n}(u, p)+\alpha_{n},\left[u_{h n}+g_{n}\right]_{-}\right] \underbrace{-\left[\varepsilon^{-1} \phi_{\delta}\left(u_{h n}+g_{n}\right),\left[u_{h n}+g_{n}\right]_{-}\right]}_{\leq 0} \\
& \leq\left[\tau_{n}(u, p)+\alpha_{n},\left[u_{h n}+g_{n}\right]_{-}-\phi_{\delta}\left(u_{h n}+g_{n}\right)\right]+\varepsilon\left[\tau_{n}(u, p)+\alpha_{n}, \varepsilon^{-1} \phi_{\delta}\left(u_{h n}+g_{n}\right)\right] \\
& \leq\left\|\tau_{n}(u, p)+\alpha_{n}\right\|_{\Gamma}\left\|\left[u_{h n}+g_{n}\right]_{-}-\phi_{\delta}\left(u_{h n}+g_{n}\right)\right\|_{\Gamma} \\
& +\varepsilon\left\|\tau_{n}(u, p)+\alpha_{n}\right\|_{M}\left\|\varepsilon^{-1} \phi_{\delta}\left(u_{h n}+g_{n}\right)\right\|_{M^{\prime}} \\
& \leq C_{*}\left[\delta+\varepsilon\left(1+\frac{h}{\varepsilon}\right)\right] \leq C_{*}(\delta+\varepsilon+h) \text {. }
\end{aligned}
$$

Summing up those estimates, we obtain

$$
\begin{aligned}
C\left\|I_{h} u-u_{h}\right\|_{1}^{2} & \leq a\left(I_{h} u-u_{h}, I_{h} u-u_{h}\right) \\
& =a\left(I_{h} u-u, I_{h} u-u_{h}\right)+a\left(u-u_{h}, I_{h} u-u_{h}\right) \\
& =a\left(I_{h} u-u, I_{h} u-u_{h}\right)+J_{1}\left(I_{h} u-u_{h}\right)+J_{2}\left(I_{h} u-u_{h}\right) \\
& \leq C_{* *} h\left\|I_{h} u-u_{h}\right\|_{1}+C_{* *} h^{2}+C_{* *} h\left\|I_{h} u-u_{h}\right\|_{1}+C_{* *} h+C_{*}(\delta+\varepsilon+h) .
\end{aligned}
$$

Therefore, we deduce

$$
\left\|I_{h} u-u_{h}\right\|_{1} \leq C_{* *}(\sqrt{h}+\sqrt{\varepsilon}+\sqrt{\delta})
$$

and

$$
\left\|u-u_{h}\right\|_{1} \leq\left\|u-I_{h} u\right\|_{1}+\left\|I_{h} u-u_{h}\right\|_{1} \leq C_{* *}(\sqrt{h}+\sqrt{\varepsilon}+\sqrt{\delta}) .
$$

This, together with (75), implies (71).

Step 2. We derive an estimation for $\left|k_{h}-k\right|$ by using (71). For sufficiently small $\varepsilon, h, \delta$ with $h \leq c_{1} \varepsilon$, according to (71) and (A2), there exists $\Gamma_{0} \subset \Gamma$ with $\left|\Gamma_{0}\right|>0$ such that $u_{n}+g_{n}>0$ and $u_{h n}+g_{n}>0$ on $\Gamma_{0}$. As in the proof of Theorem 10 (see (64)), we take $\tilde{\mu} \in C_{0}^{\infty}(\Gamma)$ satisfying $\tilde{\mu} \geq 0, \tilde{\mu} \not \equiv 0$ in $\Gamma$ and $\operatorname{supp} \tilde{\mu} \subset \Gamma_{0}$. Then, setting $\tilde{\mu}_{h}=i_{h} \mu \in M_{h}$, we have

$$
\tilde{\mu}_{h} \geq 0, \tilde{\mu}_{h} \not \equiv 0 \text { in } \Gamma, \quad \tilde{\mu}_{h}=0 \text { in } \Gamma \backslash\left(\Gamma_{0}\right), \quad\left\|\tilde{\mu}_{h}\right\|_{M} \leq C, \quad\left|\int_{\Gamma} \tilde{\mu}_{h} d \Gamma-\int_{\Gamma} \tilde{\mu} d \Gamma\right| \leq C h^{2} .
$$

Since $u_{n}+g_{n}>0$ on $\Gamma_{0}$, we have $\tau_{n}(u, p)+\alpha_{n}=0$ on $\Gamma_{0}$ in view of (22e). Substituting $\tilde{v}_{h}=\mathcal{E}_{n h} \tilde{\mu}_{h} \in$ $V_{h} \subset V$ into (72) and using (22d), we have

$$
\begin{aligned}
k \int_{\Gamma} \tilde{\mu}_{h} d \Gamma & =a\left(u, \tilde{v}_{h}\right)+b\left(\hat{p}, \tilde{v}_{h}\right)-\left(f, \tilde{v}_{h}\right)+\left[\left[\alpha, \tilde{v}_{h}\right]\right]-\int_{\Gamma}\left(\tau_{n}(u, p)+\alpha_{n}\right) \tilde{\mu}_{h} d \Gamma \\
& =a\left(u, \tilde{v}_{h}\right)+b\left(\hat{p}, \tilde{v}_{h}\right)-\left(f, \tilde{v}_{h}\right)+\left[\left[\alpha, \tilde{v}_{h}\right]\right] .
\end{aligned}
$$

This, together with (65) and (75), gives

$$
\begin{aligned}
\left|k_{h}-k\right| & \leq\left|a\left(u_{h}-u, \tilde{v}_{h}\right)\right|+\left|b\left(\hat{p}_{h}-\hat{p}, \tilde{v}_{h}\right)\right| \\
& \leq C\left(\left\|u_{h}-u\right\|_{1}+\left\|\hat{p}_{h}-\hat{p}\right\|\right) \leq C_{* *}\left(\left\|I_{h} u-u_{h}\right\|_{1}+h\right) .
\end{aligned}
$$

Step 3. We proceed to the proof of (68) and (69). We will prove

$$
J_{2}\left(I_{h} u-u_{h}\right)+\frac{\varepsilon}{2}\|\lambda\|_{\Gamma}^{2} \leq C_{* *}\left[h^{2}+\frac{h^{3}}{\varepsilon}+\varepsilon h+\frac{\delta^{2}}{\varepsilon}+(\varepsilon+h)\left\|I_{h} u-u_{h}\right\|_{1}\right],
$$


where

$$
\lambda=\tau_{n}(u, p)+\alpha_{n}-\frac{1}{\varepsilon} \phi_{\delta}\left(u_{h n}+g_{n}\right) .
$$

Recall that $J_{2}\left(I_{h} u-u_{h}\right)$ is divided into $J_{21}+J_{22}$ as in (77). We have, by $(80)$ and the standard trace theorem,

$$
J_{21} \leq\left(\|\lambda\|_{M^{\prime}}+\left|k-k_{h}\right|\right)\left\|\left(I_{h} u\right)_{n}-u_{n}\right\|_{\frac{1}{2}, \Gamma} \leq C_{* *} h\left(\|\lambda\|_{M^{\prime}}+h+\left\|I_{h} u-u_{h}\right\|_{1}\right) .
$$

We derive an estimation for $\|\lambda\|_{M^{\prime}}$. First,

$$
\|\lambda\|_{M^{\prime}}=\sup _{\mu \in M}\left(\frac{\left[\lambda, \mu-\mu_{h}\right]}{\|\mu\|_{\frac{1}{2}, \Gamma}}+\frac{\left[\lambda, \mu_{h}\right]}{\|\mu\|_{\frac{1}{2}, \Gamma}}\right) \leq C\|\lambda\|_{\Gamma} \sup _{\mu \in M} \frac{\left\|\mu-\mu_{h}\right\|_{\Gamma}}{\|\mu\|_{\frac{1}{2}, \Gamma}}+\sup _{\mu \in M} \frac{\left[\lambda, \mu_{h}\right]}{\|\mu\|_{\frac{1}{2}, \Gamma}},
$$

where $\mu_{h}$ is an arbitrary element of $M_{h}$.

In order to set $\mu_{h}$ appropriately, we use Scott and Zhang's projection $\tilde{\Pi}_{h}: H^{1}(\Omega)^{d} \rightarrow Q_{h}^{d}([31])$. The projection $\tilde{\Pi}_{h}$ satisfies the same stability and interpolation error estimates as $\Pi_{h}$. For example, we have $\left\|\tilde{\Pi}_{h} v\right\| \leq C\|v\|,\left\|\tilde{\Pi}_{h} v\right\|_{1} \leq C\|v\|_{1}$ and $\left\|v-\tilde{\Pi}_{h} v\right\| \leq C h\|v\|_{1}$ (see [9, §4.8]). Particularly, we have $\left\|v-\tilde{\Pi}_{h} v\right\|_{H^{\frac{1}{2}}(\Omega)} \leq C h^{\frac{1}{2}}\|v\|_{1}$ by the interpolation. Furthermore, $\tilde{\Pi}_{h}$ preserves the boundary condition; $\tilde{\Pi}_{h} v \in V_{h}$ for $v \in V$. At this stage, for $\mu \in M$, we set

$$
\mu_{h}=\left.\left(w_{h} \cdot n\right)\right|_{\Gamma}, \quad w_{h}=\tilde{\Pi}_{h} \mathcal{E}_{n} \mu .
$$

Then, again by the standard trace theorem,

$$
\left\|\mu-\mu_{h}\right\|_{\Gamma} \leq C\left\|\mathcal{E}_{n} \mu-\tilde{\Pi}_{h} \mathcal{E}_{n} \mu\right\|_{1} \leq C h^{\frac{1}{2}}\left\|\mathcal{E}_{n} \mu\right\|_{1} \leq C h^{\frac{1}{2}}\|\mu\|_{\frac{1}{2}, \Gamma} .
$$

In view of (73),

$$
\left[\lambda, \mu_{h}\right]=a\left(u-u_{h}, w_{h}\right)+b\left(\hat{p}-\hat{p}_{h}, w_{h}\right)-\left(k-k_{h}\right) \int_{\Gamma} \mu_{h} d \Gamma
$$

and, by (75) and (80),

$$
\begin{aligned}
{\left[\lambda, \mu_{h}\right] } & \leq C\left(\left\|u-u_{h}\right\|_{1}+\left\|\hat{p}-\hat{p}_{h}\right\|+\left|k-k_{h}\right|\right)\left\|w_{h}\right\|_{1} \\
& \leq C_{* *}\left(h+\left\|I_{h} u-u_{h}\right\|_{1}\right)\|\mu\|_{\frac{1}{2}, \Gamma} .
\end{aligned}
$$

Putting those estimates together in (83), we obtain

$$
\|\lambda\|_{M^{\prime}} \leq C_{* *}\left(h^{\frac{1}{2}}\|\lambda\|_{\Gamma}+h+\left\|I_{h} u-u_{h}\right\|_{1}\right) .
$$

Substituting this into (82) and applying Schwarz's inequality, we deduce

$$
\begin{aligned}
J_{21} & \leq C_{* *}\left(h^{2}+C h^{\frac{3}{2}}\|\lambda\|_{\Gamma}+h\left\|I_{h} u-u_{h}\right\|_{1}\right) \\
& \leq C_{* *}\left(h^{2}+\frac{h^{3}}{\varepsilon}+h\left\|I_{h} u-u_{h}\right\|_{1}\right)+\frac{\varepsilon}{6}\|\lambda\|_{\Gamma}^{2} .
\end{aligned}
$$

Next, we derive a sharp estimate for $J_{22}$. According to the third equality of (78), we have

$$
J_{22} \leq\left[\lambda,\left[u_{h n}+g_{n}\right]_{-}\right]=\underbrace{\left[\lambda,\left[u_{h n}+g_{n}\right]_{-}-\phi_{\delta}\left(u_{h n}+g_{n}\right)\right]}_{=J_{221}}-\varepsilon[\lambda, \lambda]+\underbrace{\varepsilon\left[\lambda, \tau_{n}(u, p)+\alpha_{n}\right]}_{=J_{222}} .
$$

From (40b),

$$
J_{221} \leq\|\lambda\|_{\Gamma}\left\|\left[u_{h n}+g_{n}\right]_{-}-\phi_{\delta}\left(u_{h n}+g_{n}\right)\right\|_{\Gamma} \leq C \delta\|\lambda\|_{\Gamma} \leq C \frac{\delta^{2}}{\varepsilon}+\frac{\varepsilon}{6}\|\lambda\|_{\Gamma}^{2} .
$$

Using (84), we get the estimation of $J_{222}$ as follows:

$$
\begin{aligned}
J_{222} & \leq \varepsilon\|\lambda\|_{M^{\prime}}\left\|\tau_{n}(u, p)+\alpha_{n}\right\|_{\frac{1}{2}, \Gamma} \\
& \leq C_{* *} \varepsilon\left(h^{\frac{1}{2}}\|\lambda\|_{\Gamma}+h+\left\|I_{h} u-u_{h}\right\|_{1}\right) \leq C_{* *}\left(\varepsilon h+\varepsilon\left\|I_{h} u-u_{h}\right\|_{1}\right)+\frac{\varepsilon}{6}\|\lambda\|_{\Gamma}^{2} .
\end{aligned}
$$


Summing up those estimates, we obtain

$$
J_{22} \leq C_{* *}\left(\varepsilon h+\frac{\delta^{2}}{\varepsilon}+\varepsilon\left\|I_{h} u-u_{h}\right\|_{1}\right)-\frac{2 \varepsilon}{3}\|\lambda\|_{\Gamma}^{2} .
$$

Combing this with (85), we deduce (81).

Now, instead of (79), we have by (76) and (81)

$$
\begin{aligned}
C\left\|I_{h} u-u_{h}\right\|_{1}^{2} & \leq a\left(I_{h} u-u, I_{h} u-u_{h}\right)+J_{1}\left(I_{h} u-u_{h}\right)+J_{2}\left(I_{h} u-u_{h}\right) \\
& \leq C_{* *}(h+\varepsilon)\left\|I_{h} u-u_{h}\right\|_{1}+C\left(h^{2}+\frac{h^{3}}{\varepsilon}+\varepsilon h+\frac{\delta^{2}}{\varepsilon}\right)-\frac{\varepsilon}{2}\|\lambda\|_{\Gamma}^{2} .
\end{aligned}
$$

This yields

$$
\left\|I_{h} u-u_{h}\right\|_{1} \leq C_{* *}\left(h+\sqrt{\frac{h^{3}}{\varepsilon}}+\sqrt{\varepsilon h}+\sqrt{\frac{\delta^{2}}{\varepsilon}}\right) \leq C_{* *}\left(h+\varepsilon+\sqrt{\frac{\delta^{2}}{\varepsilon}}\right),
$$

since we chose as $h \leq c_{1} \varepsilon$.

Finally, we obtain (68) and (69) by combining (75), (80), (81) and (84). This completes the proof of Theorem 18.

Remark 19. In addition to the basic assumption of Theorem 18, we suppose that

$$
u_{h n}+g_{n}>0 \text { on } \Gamma \text {. }
$$

Then, for sufficiently small $h, \varepsilon, \delta$ with $c_{2} \varepsilon \leq h \leq c_{1} \varepsilon$, we have

$$
\left\|u-u_{h}\right\|_{1}+\left\|p-p_{h}\right\| \leq C_{* *} h .
$$

In particular, we do not need to choose as $\delta \leq c_{3} h^{\frac{3}{2}}$. Inequality (87) is derived by noting $J_{22}=0$ under (86).

\section{Numerical examples}

In this section, we present some results of numerical experiments to confirm our theoretical results. We prefer the original setting (4) with (6), (11) to (1) and (2). Therefore, we consider a model Stokes problem with a nonlinear Robin condition as

$$
\begin{array}{ll}
-\nu \Delta v+\nabla q=f, \quad \nabla \cdot v=0 & \text { in } \Omega, \\
v=b & \text { on } S_{1}, \\
v=0 & \text { on } S_{2}, \\
\tau_{n}(v, q)=\frac{1}{\varepsilon} \phi_{\delta}\left(v_{n}\right), \quad v_{T}=0 & \text { on } \Gamma,
\end{array}
$$

where $\phi_{\delta}$ represents the regularized function defined as (3).

Remark 20. As described in Introduction, we are interested in computing $v$ and $q$ in (4). The unknown functions $u$ and $p$ in (1) and (2) are introduced as "perturbations" of those target variables. They clarify analysis. Moreover, the reference flow $(g, \pi)$ plays an important role in theoretical considerations, although it is not readily apparent that it is always available in actual computations.

Remark 21. In (88), we take $v_{T}=0$ instead of $\tau_{T}(u)=0$ as a boundary condition for the tangential component of $v$ on $\Gamma$. See Remark 7 .

The finite element approximation for (88) reads as follows.

$\left(\mathbf{P E} \mathbf{E}_{\varepsilon, \delta, h}^{\prime}\right)$ Find $\left(v_{h}, q_{h}\right) \in W_{h} \times Q_{h}$ such that $v_{h}=i_{h} b$ on $S_{1}$ and

$$
\begin{array}{ll}
a\left(v_{h}, w_{h}\right)+b\left(q_{h}, w_{h}\right)-\frac{1}{\varepsilon} \int_{\Gamma} \phi_{\delta}\left(v_{n}\right) w_{h n} d \Gamma=\left(f, w_{h}\right) & \left(\forall w_{h} \in V_{h}\right), \\
b\left(r_{h}, v_{h}\right)=0 & \left(\forall r_{h} \in Q_{h}\right),
\end{array}
$$


where

$$
W_{h}=\left\{v_{h} \in C^{0}(\bar{\Omega}) \mid v_{h}=0 \text { on } S_{2}, v_{h T}=0 \text { on } \Gamma,\left.v_{h}\right|_{T} \in\left[\mathcal{P}_{1}^{(d)} \oplus \operatorname{span}\left\{\varphi_{T}\right\}\right]^{d}\left(\forall T \in \mathcal{T}_{h}\right)\right\} .
$$

First, we deal with a simple example, setting $\Omega=\{(x, y) \mid 0 \leq x \leq L,-R \leq y \leq R\}, S_{1}=$ $\{0\} \times[-R, R]$, and $\Gamma=\{L\} \times[-R, R]$, we impose

$$
b(x, y)=\left(C_{0}\left(R^{2}-y^{2}\right), 0\right), \quad f \equiv 0
$$

with $C_{0}>0$. Then, (88) has the exact solution, which is given explicitly as

$$
v(x, y)=\left(C_{0}\left(R^{2}-y^{2}\right), 0\right), \quad q(x, y)=2 \nu C_{0} L\left(1-\frac{x}{L}\right) .
$$

This is the well-known Poiseuille flow.

Details of our computation are the following. Set $L=15, R=5, \nu=1 / 50$, and $C_{0}=5 /(\nu L)$. For the triangulation of $\Omega$, we use a uniform mesh composed of $12 N^{2}$ congruent right-angle triangles; The rectangle is divided into $3 N \times 2 N$ squares. Then, each small square is decomposed into two equal triangles by a diagonal. Consequently, $h=\sqrt{2} / N$. Since we have employed the $C^{1}$ regularization $\phi_{\delta}$, Newton's method is available for computing the nonlinear equation $\left(\mathrm{PE}_{\varepsilon, \delta, h}^{\prime}\right)$. Penalty parameters are chosen as $\varepsilon=\delta=h / 20$. Hence, it is ensured by Theorem 18 and Remark 19 that

$$
\left\|v-v_{h}\right\|_{1}+\left\|q-q_{h}\right\| \leq C h .
$$

To verify this point, we set

$$
E_{h}^{(1)}=\left\|v-v_{h}\right\|, \quad E_{h}^{(2)}=\left\|v-v_{h}\right\|_{1}, \quad E_{h}^{(3)}=\left\|q-q_{h}\right\|,
$$

and observe that

$$
\rho_{h}^{(i)}=\frac{\log E_{h^{\prime}}^{(i)}-\log E_{h}^{(i)}}{\log h^{\prime}-\log h} \quad(i=1,2,3)
$$

with $h^{\prime} \approx 2 h$.

The result is reported in Tab. 1 and support our theoretical result (91). We were unable to derive the $L^{2}$ error for $v_{h}$. From Tab. 1, we observe that second-order convergence actually occurs.

\begin{tabular}{l|lr|lr|lr}
\multicolumn{1}{l|}{${ }^{(1)}$} & $E_{h}^{(1)}$ & $\rho_{h}^{(1)}$ & $E_{h}^{(2)}$ & $\rho_{h}^{(2)}$ & $E_{h}^{(3)}$ & $\rho_{h}^{(3)}$ \\
\hline \hline 1.0743 & 13.9 & - & $1.20 \cdot 10^{2}$ & - & $2.07 \cdot 10^{-1}$ & - \\
0.5371 & 3.47 & 2.001 & $5.96 \cdot 10^{1}$ & 1.010 & $6.57 \cdot 10^{-2}$ & 1.656 \\
0.2685 & 0.87 & 2.000 & $2.97 \cdot 10^{1}$ & 1.003 & $2.18 \cdot 10^{-2}$ & 1.594 \\
0.1342 & 0.21 & 2.000 & $1.48 \cdot 10^{1}$ & 1.001 & $7.42 \cdot 10^{-3}$ & 1.553 \\
0.0665 & 0.052 & 2.000 & 7.17 & 1.000 & $2.56 \cdot 10^{-3}$ & 1.527
\end{tabular}

Table 1: Numerical convergence rates of $\left(\mathrm{PE}_{\varepsilon, \delta, h}^{\prime}\right)$ for $(90)$.

Next, we consider a two-dimensional branched pipe as portrayed in Fig. 2. Since this $\Omega$ is not a polygon, we approximate it by a polygon $\Omega_{h}$ with vertices located on $\partial \Omega$. On $S_{1}$, we impose a parabolic inflow similarly to (89). Fig. 3 shows the state of a numerical flow velocity $v_{h}$.

As before, we observe $\rho_{h}^{(1)}, \rho_{h}^{(2)}$ and $\rho_{h}^{(3)}$. Since, in this case, we are unable to obtain the (explicit) exact solution, we use numerical solutions with extra fine mesh. Tab. 2 presents the results. We observe that convergence rates of the $H^{1}$ error for $v_{h}$ and the $L^{2}$ error for $q_{h}$ are close to unity even in the curved domain. Moreover, that of the $L^{2}$ error for $v_{h}$ is close to 2.

Finally, we examine the convergence in terms of the penalty parameter $\varepsilon$. We now consider a non-trivial external force $f$ and the resulting flow velocity is showed in Fig. 4. 


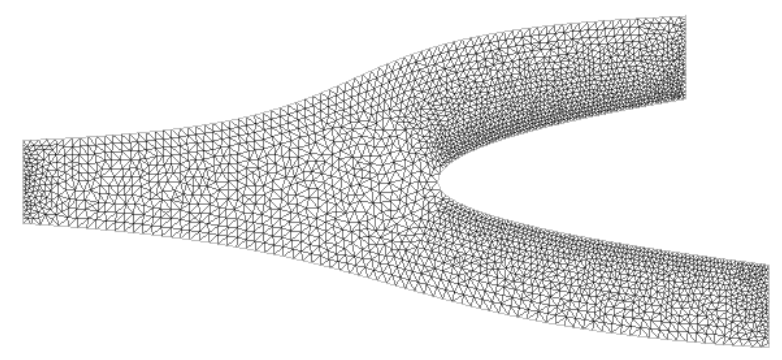

Figure 2: A branched pipe and an example of triangulation.
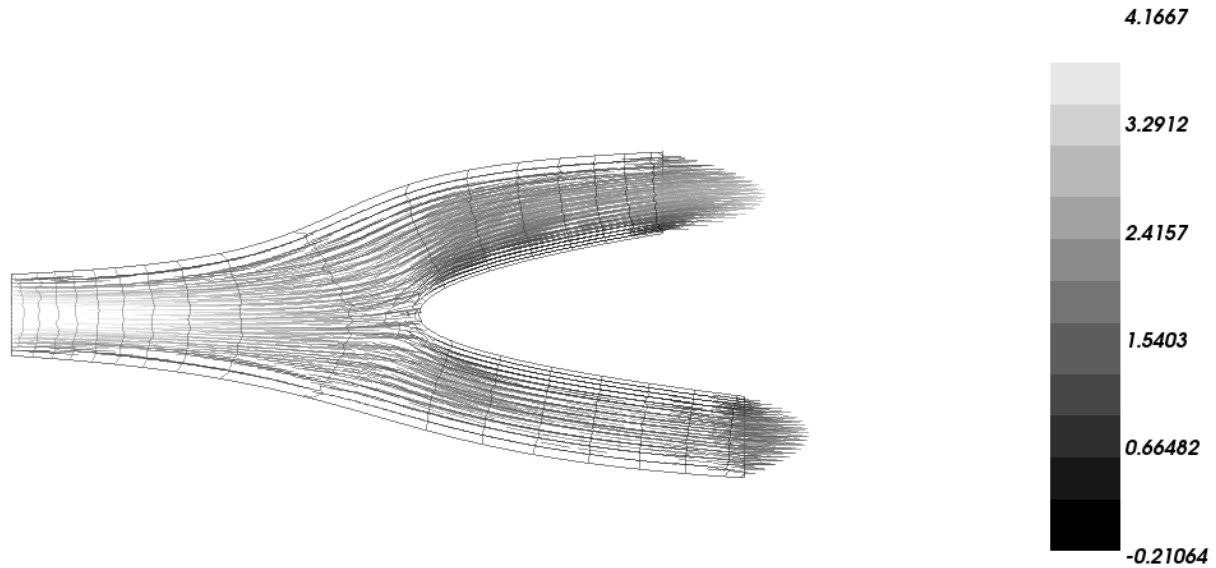

Figure 3: Velocity and pressure field in branched pipe.

Letting $\delta=\varepsilon^{\frac{3}{2}}$ (see Theorem 18), we set

$$
E_{\varepsilon}^{(1)}=\left\|v-v_{h}\right\|, \quad E_{\varepsilon}^{(2)}=\left\|v-v_{h}\right\|_{1}, \quad E_{\varepsilon}^{(3)}=\left\|q-q_{h}\right\|,
$$

and observe

$$
\rho_{\varepsilon}^{(i)}=\frac{\log E_{\varepsilon^{\prime}}^{(i)}-\log E_{\varepsilon}^{(i)}}{\log \varepsilon^{\prime}-\log \varepsilon} \quad(i=1,2,3),
$$

where $\varepsilon^{\prime} \approx 2 \varepsilon$ and $(v, q)$ is the numerical solution with extra small $\varepsilon$.

Results are reported in Tab. 3 and 4, respectively, for $h=0.6928$ and $h=0.1757$. We observe from these tables that the first order convergence with respect to $\varepsilon$ actually occurs.

\section{Acknowledgement}

We thank Professors K. Takizazawa and H. Suito, who brought the subject to our attention. We thank Dr. T. Kashiwabara for his valuable suggestions. We also thank the anonymous reviewers for their valuable comments and suggestions to improve the quality of the paper. This work is supported by JST, CREST, and JSPS KAKENHI Grant Number 23340023. 


\begin{tabular}{l|lc|lc|ll}
$h$ & $E_{h}^{(1)}$ & $\rho_{h}^{(1)}$ & $E_{h}^{(2)}$ & $\rho_{h}^{(2)}$ & $E_{h}^{(3)}$ & $\rho_{h}^{(3)}$ \\
\hline \hline 0.69279 & $2.497 \cdot 10^{-1}$ & - & 5.941 & - & $1.786 \cdot 10^{-1}$ & - \\
0.33353 & $7.767 \cdot 10^{-2}$ & 1.552 & 3.359 & 0.780 & $5.909 \cdot 10^{-2}$ & 1.513 \\
0.17571 & $2.044 \cdot 10^{-2}$ & 2.083 & 1.768 & 1.001 & $3.069 \cdot 10^{-2}$ & 1.022
\end{tabular}

Table 2: Numerical convergence rates of $\left(\mathrm{PE}_{\varepsilon, \delta, h}^{\prime}\right)$ for branched pipe.
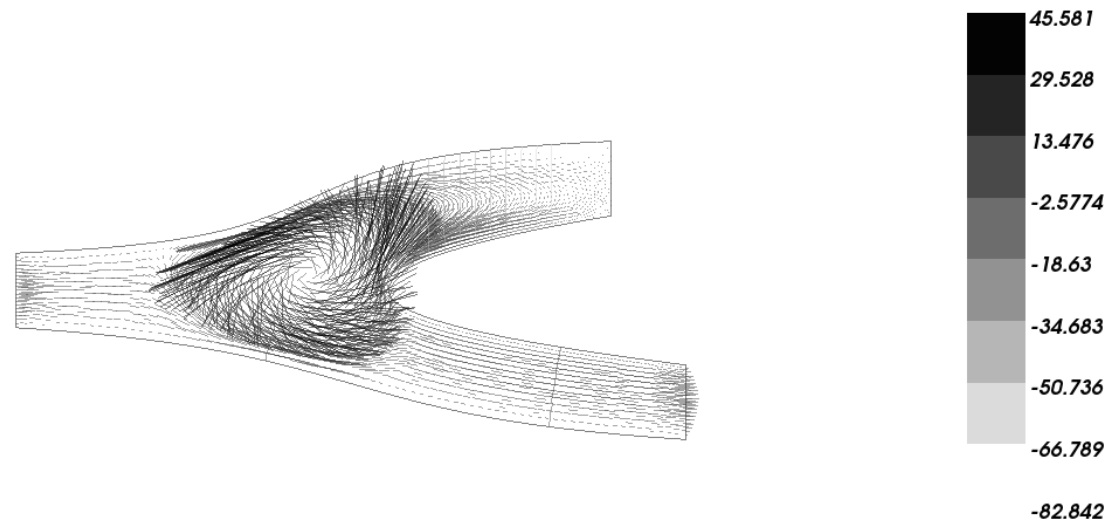

Figure 4: Velocity and pressure fields.

\section{References}

[1] M. Ayadi, L. Baffico, M. K. Gdoura, , and T. Sassi. Error estimates for stokes problem with Tresca friction conditions. ESAIM Math. Model. Numer. Anal., 48:1413-1429, 2014.

[2] C. Baiocchi and A. Capelo. Variational and Quasivariational Inequalities. Applications to Free Boundary Problems. Wiley, 1984.

[3] Y. Bazilevs, J. R. Gohean, T. J. R. Hughes, R. D. Moser, and Y. Zhang. Patient-specific isogeometric fluid-structure interaction analysis of thoracic aortic blood flow due to implantation of the Jarvik 2000 left ventricular assist device. Comput. Methods Appl. Mech. Engrg., 198:3534-355, 2009.

[4] F. Ben Belgacem, Y. Renard, and L. Slimane. A mixed formulation for the signorini problem in nearly incompressible elasticity. Appl. Numer. Math., 54:1-22, 2005.

[5] D. Boffi, F. Brezzi, and M. Fortin. Mixed Finite Element Methods and Applications. Springer, 2013.

[6] M. Boman. Estimates for the $L_{2}$-projection onto continuous finite element spaces in a weighted $L_{p}$-norm. BIT Numer. Math., 46:249-260, 2006.

[7] F. Boyer and P. Fabrie. Outflow boundary conditions for the incompressible non-homogeneous NavierStokes equations. Discrete Contin. Dyn. Syst. Ser. B, 7:219-250, 2007.

\begin{tabular}{l|lr|lc|ll}
$\varepsilon$ & $E_{\varepsilon}^{(1)}$ & $\rho_{\varepsilon}^{(1)}$ & $E_{\varepsilon}^{(2)}$ & $\rho_{\varepsilon}^{(2)}$ & $E_{\varepsilon}^{(3)}$ & $\rho_{\varepsilon}^{(3)}$ \\
\hline \hline 0.034639 & 7.315 & - & 22.400 & - & 9.359 & - \\
0.017319 & 3.649 & 1.003 & 11.177 & 1.002 & 4.668 & 1.003 \\
0.008659 & 1.749 & 1.060 & 5.358 & 1.060 & 2.238 & 1.060
\end{tabular}

Table 3: Numerical convergence rate of $\left(\mathrm{PE}_{\varepsilon, \delta, h}^{\prime}\right)$ for $h=0.6928$. 


\begin{tabular}{l|lc|lc|lc}
$\varepsilon$ & $E_{\varepsilon}^{(1)}$ & $\rho_{\varepsilon}^{(1)}$ & $E_{\varepsilon}^{(2)}$ & $\rho_{\varepsilon}^{(2)}$ & $E_{\varepsilon}^{(3)}$ & $\rho_{\varepsilon}^{(3)}$ \\
\hline \hline 0.0087854 & 2.088 & - & 6.421 & - & 2.469 & - \\
0.0043927 & 1.024 & 1.028 & 3.149 & 1.027 & 1.210 & 1.028 \\
0.0021964 & 0.486 & 1.073 & 1.497 & 1.072 & 0.575 & 1.073
\end{tabular}

Table 4: Numerical convergence rate of $\left(\mathrm{PE}_{\varepsilon, \delta, h}^{\prime}\right)$ for $h=0.1757$.

[8] F. Boyer and P. Fabrie. Mathematical Tools for the Study of the Incompressible Navier-Stokes Equations and Related Models. Springer, 2013.

[9] S. C. Brenner and L. R. Scott. The Mathematical Theory of Finite Element Methods. Springer, 2008.

[10] H. Brezis. Functional Analysis, Sobolev Spaces and Partial Differential Equations. Springer, 2011.

[11] C. H. Bruneau and P. Fabrie. Effective downstream boundary conditions for incompressible NavierStokes equations. Internat. J. Numer. Methods Fluids, 19:693-705, 1994.

[12] C. H. Bruneau and P. Fabrie. New efficient boundary conditions for incompressible Navier-Stokes equations: a well-posedness result. RAIRO Modél. Math. Anal. Numér., 30:815-840, 1996.

[13] F. Chouly and P. Hild. On convergence of the penalty method for unilateral contact problems. Appl. Numer. Math., 65:27-40, 2013.

[14] M. Crouzeix and V. Thomée. The stability in $L_{p}$ and $W_{p}^{1}$ of the $L_{2}$-projection onto finite element function spaces. Math. Comp., 48:521-532, 1987.

[15] G. Drouet and P. Hild. Optimal convergence for discrete variational inequalities modelling signorini contact in $2 \mathrm{~d}$ and $3 \mathrm{~d}$ without additional assumptions on the unknown contact set. SIAM J. Numer. Anal., 53:27-40, 2015.

[16] L. Formaggia, A. Quarteroni, and A. Veneziani. Cardiovascular Mathematics. Springer, 2009.

[17] V. Girault and P. A. Raviart. Finite Element Methods for Navier-Stokes Equations. Springer, 1986.

[18] P. M. Gresho and R. L. Sani. Incompressible Flow and the Finite Element Method, Volume 2, Isothermal Laminar Flow. Wiley, 2000.

[19] P. Grisvard. Behavior of the solutions of an elliptic boundary value problem in a polygonal or polyhedral domain. Numerical Solution of P.D.E's III, Proc. Third Sympos. (SYNSPADE), pages 207-274, 1976.

[20] P. Grisvard. Elliptic Problems in Nonsmooth Domains. Pitman, 1985.

[21] J. G. Heywood, R. Rannacher, and S. Turek. Artificial boundaries and flux and pressure conditions for the incompressible Navier-Stokes equations. Internat. J. Numer. Methods Fluids, 22:325-352, 1996.

[22] J. Douglas Jr., T. Dupont, and L. Wahlbin. The stability in $L^{q}$ of the $L^{2}$-projection into finite element function spaces. Numer. Math., 23:193-197, 1975.

[23] T. Kashiwabara. Finite element method for Stokes equations under leak boundary condition of friction. SIAM J. Numer. Anal., 51:2448-2469, 2013.

[24] N. Kikuchi and J. T.Oden. Contact Problems in Elasticity. SIAM, 1988.

[25] Y. Li and R. An. Semi-discrete stabilized finite element methods for Navier-Stokes equations with nonlinear slip boundary conditions based on regularization procedure. Numer. Math., 117:1-36, 2011.

[26] Y. Li and R. An. Penalty finite element method for Navier-Stokes equations with nonlinear slip boundary conditions. Internat. J. Numer. Methods Fluids, 69:550-576, 2012. 
[27] J. L. Lions. Quelques Méthodes de Résolution des Problèmes aux Limites Non Linéaires. Dunod, 1969.

[28] J. L. Lions. Some remarks on variational inequalities. Proc. Internat. Conf. on Functional Analysis and Related Topics, pages 269-282, 1969.

[29] J. L. Lions and E. Magenes. Non-homogeneous Boundary Value Problems and Applications, I. Springer, 1972.

[30] N. Saito and H. Fujita. Remarks on traces of $H^{1}$-functions defined in a domain with corners. J. Math. Sci. Univ. Tokyo, 7:325-345, 2000.

[31] L. R. Scott and S. Zhang. Finite element interpolation of non-smooth functions satisfying boundary conditions. Math. Comp., 54:483-493, 1990.

[32] C. A. Taylor, T. J. Hughes, and C. K. Zarins. Finite element modeling of blood flow in arteries. Comput. Methods Appl. Mech. Engrg., 158:155-196, 1998.

[33] R. Temam. Navier-Stokes Equations: Theory and Numerical Analysis. AMS, AMS Chelsea Publishing, 2001.

[34] G. Zhou and N. Saito. The Navier-Stokes equations under a unilateral boundary condition of Signorini's type. http://www.ms.u-tokyo.ac.jp/preprint/2015/, 2015. 